\title{
Research Article \\ circRNA Expression Profile in Dental Pulp Stem Cells during Odontogenic Differentiation
}

\author{
Ming Chen, ${ }^{1,2}$ Yeqing Yang, ${ }^{1,2}$ Junkai Zeng, ${ }^{2,3}$ Zilong Deng, ${ }^{2,3}$ and Buling Wu $\mathbb{D}^{2,3}$ \\ ${ }^{1}$ Stomatological Hospital, Southern Medical University, Guangzhou, China \\ ${ }^{2}$ School of Stomatology, Southern Medical University, Guangzhou, China \\ ${ }^{3}$ Nanfang Hospital, Southern Medical University, Guangzhou, China \\ Correspondence should be addressed to Buling Wu; bulingwu@smu.edu.cn
}

Received 17 December 2019; Revised 25 June 2020; Accepted 8 August 2020; Published 1 September 2020

Academic Editor: Sumanta Chatterjee

Copyright (c) 2020 Ming Chen et al. This is an open access article distributed under the Creative Commons Attribution License, which permits unrestricted use, distribution, and reproduction in any medium, provided the original work is properly cited.

Introduction. Odontogenic differentiation of human dental pulp stem cells (hDPSCs) is a key step of pulp regeneration. Recent studies showed that circular RNAs (circRNAs) have many biological functions and that competing endogenous RNA (ceRNA) is their most common mechanism of action. However, the role of circRNAs in hDPSCs during odontogenesis is still unclear. Methods. Isolated hDPSCs were cultured in essential and odontogenic medium. Total RNA was extracted after 14 days of culture, and then, microarray analysis was performed to measure the differential expressions of circRNAs. Quantitative reverse transcription-polymerase chain reaction (qRT-PCR) was then performed to validate the microarray results. Based on microarray data from this study and available in the database, a ceRNA network was constructed to investigate the potential function of circRNAs during odontogenesis. In addition, Gene Ontology (GO) and Kyoto Encyclopedia of Genes and Genomes (KEGG) analyses were performed to investigate the potential correlation between signaling pathways and circRNAs. In addition, qRTPCR and Western blot analysis were used to explore the function of hsa_circRNA_104101. Results. We found 43 upregulated circRNAs and 144 downregulated circRNAs during the odontogenic differentiation process (fold change $>1.5$ and $<-1.5$, respectively; $P<0.05)$. qRT-PCR results were in agreement with the microarray results. Bioinformatic analysis revealed that the Wnt signaling pathway and the TGF- $\beta$ signaling pathway, as well as the other pathways associated with odontogenic differentiation, were correlated to the differentially expressed circRNAs. hsa_circRNA_104101 was proved to promote the odontogenic differentiation of hDPSCs. Conclusion. This study reported 187 circRNAs that were differentially expressed in hDPSCs during odontogenic differentiation. Bioinformatic analysis of the expression data suggested that circRNA-miRNAmRNA networks might act as a crucial mechanism for hDPSC odontogenic differentiation, providing a theoretical foundation for the study of pulp regeneration regulation by circRNAs.

\section{Introduction}

Pulpal and periapical diseases are the most common oral diseases: the traditional treatment of choice is root canal therapy. However, the excessive loss of pulp tissue could increase the risks of fracture and the subsequent failure of the root canal therapy, resulting in serious adverse effects, including intraradicular microorganisms, extraradicular infection, foreign body reaction, and true cysts [1]. Thanks to the advancements of tissue engineering, regenerative medicine, and material science, pulp regeneration will become the new desirable stan- dard, particularly the regeneration of the dentin-pulp complex with biological techniques [2]. The regeneration requires three essential prerequisites: seed cells, cytokines, and scaffolds [3, 4]. In recent years, a variety of stem cells have been isolated from oral tissues, such as human dental pulp stem cells (hDPSCs) [5], stem cells from apical papilla (SCAPs) [6], periodontal ligament stem cells (PDLSCs) [7], dental follicle progenitor cells (DFPCs) [8], and stem/progenitor cells isolated from the human pulp of exfoliated deciduous teeth (SHED) [9]. hDPSCs are the most common dental-derived stem cells used in the study of odontogenic differentiation [10]: they originate 
from neural crest mesenchymal stem cells and play an important role in pulp regeneration, thanks to their multiple differentiation potential and self-renewal capacity [11, 12]. A previous study reported the differentiation of pulp-like tissues in a subcutaneous immunodeficient mouse model, after the implantation of human tooth root segments with 3D-DPSCs [2]. Although many groups have been studying the odontogenic differentiation [13-15], the exact molecular mechanisms are still poorly understood, and thus, the regeneration of dentin-pulp complex in the clinical context is still not implemented [16]. Therefore, the search for new biomarkers linked to the molecular mechanisms of odontogenic differentiation is extremely important for studying pulp regeneration.

In recent years, research on noncoding RNA (ncRNA) has gradually become more prominent. Circular RNA (circRNA) is a family of ncRNA characterized by a cyclic covalent structure. Compared with linear RNA, its cyclic structure confers resistance to RNase $\mathrm{R}$ digestion $[17,18]$. Thus, circRNAs are not easily degraded and are more stable than linear RNA [19]. In addition, circRNAs are widely distributed in the organism and have a high evolutionary conservation and tissue cell expression specificity [20]. In some cases, the abundance of circRNA exceeds the abundance of the related linear mRNA by a factor of 10 [21, 22]. Therefore, circRNAs are considered ideal diagnostic markers and therapeutic targets, with very important research significance and clinical value [21, 22].

Some studies have found that microRNAs (miRNAs) may act as negative regulators of mRNA translation through the binding to the $3^{\prime}$-UTR mRNA region. Intriguingly, thanks to the presence of miRNA binding sites, circRNAs can function as competing endogenous RNA (ceRNA) by sequestering the relative miRNAs, inhibiting their function [23]. It has been already reported that circRNAs play an important role in the regulation of pluripotent dental stem cell differentiation, but these studies were focused on oral maxillofacial tumors and periodontal diseases [24, 25]. Moreover, a previous research found that the circRNA CDRlas promoted the osteoblastic differentiation of periodontal ligament stem cells by downregulating miR-7 and concurrently stimulating the expression of GDF5, which is usually inhibited by miR-7. GDF5 then activates the Smad1/5/8 and p38 MAPK differentiation pathway [26]. In the same year, Wang et al. found that the circRNA DOCK1, functioning as ceRNA, can regulate the expression of BIRC3, a protein involved in apoptosis in oral squamous cell carcinomas (OSCC) [25]. In addition, Li et al. suggested that the circRNA circNTATC1 could promote the osteogenic differentiation of apical papilla stem cells. By using CircBase, they predicted that the miRNA miR-4483 contains binding sites for circNFATC1 and that the expression of circNFATC1 is negatively correlated with miR-4483 expression [27]. However, now, the effects of circRNAs on hDPSC odontogenic differentiation are unclear. Thus, studying the molecular mechanisms of circRNA function can provide new biomarkers and a potential target for the regenerative treatment using mesenchymal stem cells.

In this paper, we analyzed the differential expression of circRNAs in hDPSCs during odontogenic differentiation by using microarray technology. Then, we validated the top 6 differentially expressed circRNAs by qRT-PCR. Using available data on miRNAs, TargetScan, miRDB, CircBase, CircBank, and other databases, we performed a bioinformatic analysis to construct a circRNA-miRNA-mRNA network of odontogenic gene regulation. Next, we performed Gene Ontology (GO) and Kyoto Encyclopedia of Genes and Genomes (KEGG) analyses to detect the association between circRNA differential expression and pathways associated with odontogenic differentiation. Finally, hsa_circRNA_104101 was selected for further research according to the bioinformatic analyses, and it might be involved in the process of odontogenic differentiation of hDPSCs through sponge effect. Our study was aimed at providing a theoretic foundation for elucidating the circRNA molecular mechanism of hDPSCs during odontogenic differentiation.

\section{Materials and Methods}

2.1. Cell Culture and Identification. hDPSCs were isolated from the teeth acquired from 18-25-year-old patients who have undergone tooth extraction at the Department of Stomatology of Nanfang Hospital, Southern Medical University, Guangzhou, Guangdong, China. All experimental protocols were approved by the Ethical Committee of Southern Medical University. Culturing programs of hDPSCs were the same as described previously [28]. Then, hDPSCs were cultured in Dulbecco's modified Eagle's medium (DMEM) added with $10 \%$ fetal bovine serum (FBS; GIBCO, Life Technologies, Australia), $100 \mathrm{U} / \mathrm{ml}$ penicillin, and $100 \mu \mathrm{g} / \mathrm{ml}$ streptomycin (Sigma, St. Louis, Mo, USA) at $37^{\circ} \mathrm{C}$ under $5 \% \mathrm{CO}_{2}$ in air. The culture media were renewed every 3 days. hDPSCs of passages 3-5 were used in follow-up experiments [29, 30], respectively, cultured in essential and odontogenic medium. hDPSCs in the noninduced were cultured in 10\% FBS in DMEM with no supplements. hDPSCs in the induced group were cultured with an odontogenic differentiation medium containing $50 \mathrm{mg} / \mathrm{ml}$ ascorbic acid, $100 \mathrm{nmol} / \mathrm{l}$ dexamethasone, and $10 \mathrm{mmol} / \mathrm{l} \beta$-glycerophosphate (Sigma, St Louis, Mo, USA) in DMEM for 14 days $[31,32]$. The culture media were renewed every 3 days.

Odontogenic, adipogenic, and chondrogenic differentiation was performed to identify the multiple differentiation potential of hDPSCs. Alizarin red, oil red O, and Alcian blue staining is commonly used to verify the formation of calcified nodules, lipid droplets, and mucopolysaccharides after stem cell osteogenic, adipogenic, and chondrogenic differentiation [32-34]. When hDPSCs were in the 3rd generation, logarithmic growth phase, we removed the complete original medium, washed cells with PBS buffer for 2-3 times, and digested them with $0.25 \%$ trypsin to collect the cell suspension and then inoculated into 6 -well plates, $1 \times 10^{5}$ cells/well. The fresh medium was replaced every 3 days. When the cells proliferated to a density of $80 \%$, the cells were replaced with odontogenic (contained with 10\% FBS, $50 \mathrm{mg} / \mathrm{ml}$ ascorbic acid, $100 \mathrm{nmol} / \mathrm{l}$ dexamethasone, and $10 \mathrm{mmol} / \mathrm{l} \quad \beta$-glycerophosphate), adipogenic (contained with $10 \%$ FBS, $0.5 \mathrm{mM}$ isobutylmethylxanthine (IBMX), 10-6M dexamethasone, $10 \mu \mathrm{g} / \mathrm{ml}$ insulin, and $200 \mu \mathrm{M}$ indomethacin), and chondrogenic 
(contained with 10\% FBS, $1 \%$ ITS+Premix, $0.1 \mu \mathrm{M}$ dexamethasone, $0.2 \mathrm{mM} \mathrm{L}$-ascorbic acid-2-phosphate, $1 \%$ pen/strep, and $10 \mathrm{ng} / \mathrm{ml}$ TGF- $\beta 3$ ) induction medium, and the cells were placed in a $37^{\circ} \mathrm{C}, 5 \% \mathrm{CO}_{2}$ cell culture incubator. The culture media were renewed every 3 days.

Flow cytometry was used to identify the cell phenotypes of hDPSCs. hDPSCs at the 3rd passage were isolated by trypsin, adjusted to a concentration of $1 \times 10^{6} / \mathrm{ml} /$ well. The cells were conjugated for surface markers of hematopoietic cells (CD45, CD34) and mesenchymal stem cells (Stro-1, CD90, CD44, and CD29) according to protocol and then were detected by flow cytometry. hDPSCs at the 3rd passage with a concentration of 100 cells/well were inoculated into 6-well plates and cultured for 14 days. After that, the cells were fixed with $4 \%$ paraformaldehyde and stained with crystal violet stain.

2.2. RNA Extraction. Total RNAs were extracted from induced and noninduced hDPSCs with a TRIzol reagent (Invitrogen, Life Technologies) based on the manufacturer's protocol. Total RNA was quantified by NanoDrop ND1000 (Thermo Scientific, USA).

2.3. $\operatorname{circRNA}$ Microarray. After extraction and purification, the total RNAs of the induced and noninduced groups (three cell samples per group) were analyzed by using microarray. Arraystar Human circRNA Array (Arraystar, USA) was used to perform RNA expression profiling. After RNAs were digested with $\mathrm{RNase} \mathrm{R}$, a random priming method (Arraystar Super RNA Labeling Kit; Arraystar) was used for the amplification and transcription of circRNAs to transfer into fluorescent cRNA. The arrays were analyzed by the Agilent Scanner G2505C, and Agilent Feature Extraction software (version 11.0.1.1) was used to access obtained array images.

2.4. Bioinformatic Analysis. In order to understand the potential functions of the differentially expressed circRNAs that were statistically significant $(P<0.05$; fold change $>1.5$ or <-1.5), miRanda and TargetScan software were used to predict the putative circRNA/miRNA interactions. Then, potential target genes of miRNAs were predicted using the TargetScan, miRDB, CircBase, and CircBank databases. Both GO analysis and KEGG were carried out. The GO project is able to represent the characterization of gene and gene product in all organisms. The ontology contains cellular component, biological process, and molecular function. To predict the pathways, KEGG was performed to sort differentially expressed genes on the basis of the KEGG database. The ceRNA networks were constructed with circRNAs, miRNAs, and mRNAs. Cytoscape 3.5.1 was used to reveal the networks of circRNA-miRNA-mRNA.

2.5. Identification of hsa_circRNA_104101. Agarose gel electrophoresis and Sanger sequencing were required to verify hsa_circRNA_104101. According to the sequence information of hsa_circRNA_104101, we designed the divergent primer and the convergent primer (Table 1). cDNA (complementary DNA) and gDNA (genomic DNA) were extracted from induced and noninduced hDPSCs based on the manufacturer's protocol. Then, cDNA and gDNA were, respectively, for PCR amplification, and the amplified prod- ucts were obtained by agarose gel electrophoresis. The PCR products of hsa_circRNA_104101 in the cDNA were sent to the company for Sanger sequencing.

2.6. Lentivirus Construction and Infection. The siRNA104101 (divided into 1, 2, and 3 strands) (Table 2) and siRNA-control were used to carry out instantaneous gene knockdown of hDPSCs using liposome as the carrier. At $72 \mathrm{~h}$ after transfection, the expression level of hsa_circRNA_ 104101 after gene knockdown was detected by qRT-PCR. Next, the second passages of hDPSCs were selected for experiments and construction of hsa_circRNA_104101 shRNA lentivirus. Finally, we need to determine the MOI of lentivirus-infected cells and the optimal infection conditions.

2.7. Alkaline Phosphatase and Alizarin Red Staining. Alkaline phosphatase (ALP) activity staining was performed using the NBT/BCIP Staining Kit (Beyotime Biotech, Shanghai, China); Alizarin red staining was showed to visualize mineral deposition in each group. Cells were washed with PBS for three times and were fixed in $4 \%$ paraformaldehyde for 15 mins. After washing, the hDPSCs were stained with the NBT/BCIP Staining Kit and Alizarin red. The results of each group were photographed under an inverted microscope.

2.8. $q R T$-PCR Validation. Total RNAs were isolated from two groups of hDPSCs. $1 \mu \mathrm{g}$ of RNA per sample was reverse transcribed into cDNA using a cDNA Reverse Transcription Kit (Takara, Tokyo, Japan). Divergent primers were used for analysis of circRNAs. qRT-PCR was performed in a $20 \mu \mathrm{L}$ of the reaction system, and glyceraldehyde-3-phosphate dehydrogenase (GAPDH) was used as an internal control on the Roche LightCycler ${ }^{\circledR} 480$ sequence detection system. All experiments were performed in triplicate, and relative gene expression was represented as fold change using the $2^{-}$ $\Delta \Delta \mathrm{Ct}$ method. A value of $P<0.05$ was considered statistically significant [35]. The sequences of gene-specific primers are listed in Table 3.

2.9. Western Blot Analysis. Total protein was isolated from hDPSCs using the RIPA lysis buffer and quantitated using the BCA assay. The protein lysates were separated by $10 \%$ SDS-PAGE, followed by transferring onto PVDF membranes. Primary antibodies against DSPP, DMP1, ALP, OCN, and GAPDH were diluted at 1:1000. After blockage in protein free rapid blocking buffer for $20 \mathrm{~min}$, the PVDF membranes were incubated with primary antibodies overnight at $4^{\circ} \mathrm{C}$. Next, PVDF membranes were incubated with corresponding secondary antibodies for $1 \mathrm{~h}$ at room temperature. Immunoreactive proteins were detected by using the ECL Kit (Beyotime Biotech, Shanghai, China), and the gray value of protein bands was calculated by ImageJ software.

2.10. Statistical Analysis. All statistical analyses were performed by using SPSS 19.0 software. Quantitative data were presented as the mean \pm standard deviation from three independent records. Student's $t$-test was used to calculate the statistical significance. $P<0.05$ was considered statistically significant. 
TABLE 1: The primer list.

\begin{tabular}{lc}
\hline Gene & Primer sequence $\left(5^{\prime}-3^{\prime}\right)$ \\
\hline GAPDH & $5^{\prime}$ CTGGGCTACACTGAGCACC3 $^{\prime}$ \\
& $5^{\prime}$ AAGTGGTCGTTGAGGGCAATG3 ${ }^{\prime}$ \\
hsa_circRNA_104101 & F: 5' GGAGCAACAGTAGAAAGTTCTCTA 3' length \\
Divergent primer & R: $5^{\prime}$ GGCACCTTCATCAGTAGTCATT 3 \\
hsa_circRNA_104101 & F: $5^{\prime}$ CATGGTAGCCACCCCAATGT 3' \\
Convergent primer & R: $5^{\prime}$ AATGGTGAGGAAACGCCGAT 3' \\
\hline
\end{tabular}

TABLE 2: The sequence information of siRNA.

\begin{tabular}{lc}
\hline Gene & siRNA sequence $\left(5^{\prime}-3^{\prime}\right)$ \\
\hline GAPDH & GCAACAGUAGAAAGUUCUCUATT \\
hsa_circRNA_104101 si-1 sense & UAGAGAACUUUCUACUGUUGCTT \\
hsa_circRNA_104101 si-1 antisense & GUAGAAAGUUCUCUACUUAAATT \\
hsa_circRNA_104101 si-2 sense & UUUAAGUAGAGAACUUUCUACTT \\
hsa_circRNA_104101 si-2 antisense & CAGUAGAAAGUUCUCUACUUATT \\
hsa_circRNA_104101 si-3 sense & UAAGUAGAGAACUUUCUACUGTT \\
hsa_circRNA_104101 si-3 antisense & GCAACAGUAGAAAGUUCUCUATT \\
\hline
\end{tabular}

TABLE 3: The primer list was used for real-time quantitative PCR.

\begin{tabular}{|c|c|c|}
\hline Gene & Forward primer & Reverse primer \\
\hline GAPDH & $5^{\prime}$ CTGGGCTACACTGAGCACC $3^{\prime}$ & $5^{\prime}$ AAGTGGTCGTTGAGGGCAATG3' \\
\hline DSPP & 5'TTTGGGCAGTAGCATGGGC3' & 5'CCATCTTGGGTATTCTCTTGCCT3' \\
\hline DMP1 & 5'CTCCGAGTTGGACGATGAGG3' & 5'TCATGCCTGCACTGTTCATTC3' \\
\hline $\mathrm{OCN}$ & 5' ACCTCACCTTCCTCTACTTGG3' & 5'TGGTGTCATTAGCCTTGCAG3' \\
\hline hsa_circRNA_104101 & 5'GGAGCAACAGTAGAAAGTTCTCTA3' & 5'GGCACCTTCATCAGTAGTCATT3' \\
\hline hsa_circRNA_406763 & 5’ GTCTTTAGTCTTGGCAAAGGTGT3' & 5'CAATGCATCAGAAAGGGAACA3' \\
\hline hsa_circRNA_002161 & 5'TCCGGAGAACCAAACGGAAA3' & $5^{\prime}$ GAACTGTCCAAAGGCGGAAAA3' \\
\hline hsa_circRNA_005044 & 5'CAAAGCAACGAGCTCTATGCC $3^{\prime}$ & 5'CAAAGCAACGAGCTCTATGCC $3{ }^{\prime}$ \\
\hline hsa_circRNA_079813 & 5'TGGAACCTACAAGCAGCACC $3^{\prime}$ & 5'TGCCATCAGGATTTCGATCTGT3' \\
\hline hsa_circRNA_008336 & 5'TGGAACCTACAAGCAGGCAT3' & 5'CTGGCAAAACCTGGGAAGAGA3' \\
\hline
\end{tabular}

\section{Results}

3.1. Identification and Odontogenic Differentiation of hDPSCs. hDPSCs extracted from dental pulp were cultured to the 3 rd passages in normal media (Figures 1(a) and 1(b)). The results of flow cytometry revealed that hDPSCs could express mesenchymal stem cell markers CD44 (99.99\%), CD29 (97.96\%), CD90 (99.99\%), and Stro-1 (18.39\%) but barely express hematopoietic cell markers including CD45 (0.46\%) and CD34 $(0.35 \%)$ (Figures $1(\mathrm{c})-1(\mathrm{~h})$ ). A single cell was obtained by limiting dilution technique, and clone formation was observed after 14 days of single cell culture (Figures 1(i) and 1(j)). Staining of Alizarin red, oil red $\mathrm{O}$, and Alcian blue was used to confirm multipotency of hDPSCs after 21 days of induction (Figures $1(\mathrm{k})-1(\mathrm{p})$ ). Subsequently, the qRT-PCR results suggested that the expressions of odontoblastic markers dentin sialophosphoprotein (DSPP), dentin matrix acid phosphoprotein 1 (DMP1), and osteocalcin (OCN) were upregulated. Our findings agree with the previous study reporting the differentiation of hDPSCs into odontoblasts.

3.2. Expression Profiles of circRNA during Odontogenic Differentiation. The expression profiles of 12929 human circRNAs of hDPSCs during odontogenic differentiation were obtained by the use of circRNA microarray. The heatmap analysis revealed that the inner-group variation was small, 
suggesting that these alterations may be common in the odontogenic differentiation of hDPSCs (Figure 2(a)). The box plot suggests that the distribution of the intensities among the whole samples was practically consistent (Figure 2(b)). As can be seen from the volcano plots, we found that compared with the noninduced group, 187 circRNAs displayed beyond a 1.5-fold differential expression in the induced group, and 44 circRNAs were upregulated while 143 circRNAs were downregulated (Figure 2(c)). The details of differentially expressed circRNAs are shown in Tables S1 and S2. Among these circRNAs with statistical significance, most of them are distributed on human chromosome2, chromosome6, and chromosome7, of which the upregulated circRNAs are mainly distributed on chromosome2, while the downregulated circRNAs are mainly distributed on chromosome6 and chr7. circRNAs can be divided into three types: exon, intron, and sense overlapping, but most of these circRNAs were mapped to exons.

3.3. qRT-PCR Validation of circRNA Expression. To confirm the results of the microarray data experiments, qRT-PCR analysis was performed on induced and noninduced hDPSCs, respectively. Compared with the noninduced group, hsa_circRNA_104101, hsa_circRNA_406763, hsa_circRNA_002161, and hsa_circRNA_005044 were upregulated by 19.03, 15.95, 8.29 , and 3.78 -fold, respectively, in the induced group. The expressions of hsa_circRNA_079813 and hsa_circRNA_ 008336 were downregulated by 3.85 and 3.74 -fold, respectively. Among the 187 circRNAs with significantly different expressions before and after mineralization induction, these 6 circRNAs showed the most significant changes, and primers with good specificity could be designed for them. Therefore, these six differentially expressed circRNAs were selected for verification, and all qRT-PCR results were in the agreement with the normalized expression of microarray data (Figures 2(d)-2(j)).

3.4. circRNA-miRNA-mRNA Network. Analysis of 187 significant differences (fold change $>1.5$ or $<-1.5, P<0.05$ ) in circRNAs based on biological information will have a number of potential regulatory mechanisms. Then, we selected several circRNAs from the circRNAs with significant differences for further research to construct circRNA-miRNAmRNA networks, including 5 circRNAs from upregulated genes and 4 from downregulated genes. First of all, miRanda and TargetScan software were used to determine the circRNAs combined with miRNAs, and TargetScan, miRDB, CircBase, and CircBank were used to predict target mRNAs. Then, we evaluated the miRNA response elements (MREs) related to circRNAs to verify all of the circRNA-miRNA connections. The miRNAs were ranked based on their mirSVR scores, and the top 5 miRNAs for each circRNA were selected for further analysis. We predicted and selected 25 corresponding target genes for each miRNA based on transcriptome from microarray results. After that, 2 ceRNA networks were built by the use of Cytoscape: one network constructs with downregulated circRNAs, miRNAs, and mRNAs (fold change $<-1.5 ; P<0.05$ ); the other one consists of upregulated circRNAs, miRNAs, and mRNAs (fold change $>1.5 ; P$ $<0.05$ ) (Figures 3(a) and 3(b)).
3.5. GO and KEGG Pathway Analysis. To investigate the biological functions of the dysregulated circRNAs, we selected the top five predicted miRNAs for each circRNA through specific base pairing. We performed GO and KEGG pathway analysis with their target genes. GO analysis results showed that the enriched GO terms for the biological process were cell communication, signal transduction, and regulation of nucleobase and nucleotide. Through cellular component analysis, the target genes were widely involved in the composition of the nucleus, cytoplasm, plasma membrane, etc. The molecular function structured networks indicate that the circRNA target genes can participate in a variety of molecular functions, including DNA binding, transporter activity, and receptor activity (Figures $4(\mathrm{a})-4(\mathrm{f})$ ). With the latest KEGG database, pathway analysis was performed to identify the biological functions of circRNAs and correlate the differentially expressed circRNAs with corresponding target genes. These differential genes were enriched in signaling pathways regulating pluripotency of stem cells, Wnt signaling pathway, TGF- $\beta$ signaling pathway, and some other signaling pathways associated with the differentiation of hDPSCs to odontogenic cells (Figures 4(g) and 4(h)).

3.6. Identification of hsa_circRNA_104101. The results of agarose gel electrophoresis showed that the amplified products of all two primers were single bands, and the bands were consistent with the primer amplification length. What is more, a set of convergent primer can amplify not only gDNA but also cDNA; divergent primer amplified circRNA in cDNA but not in gDNA (Figure 5(a)). The result of Sanger sequencing was consistent with the back-splice junction of hsa_circRNA_104101 (Figure 5(b)).

3.7. Construction of hsa_circRNA_104101 shRNA Lentivirus and Cell Infection. It was found that all three designed siRNA-104101 could downregulate the expression of hsa circRNA_104101 in hDPSCs, and the results of all siRNA were statistically significant $(P<0.01)$ (Figure $6(\mathrm{a})$ ), among which the second strand was the most downregulated with the most obvious results. According to the results of siRNA transfection, sh-circ104101 was designed with the second strand, and hDPSCs were transfected with the best infection condition (MOI:30 and HiTransG P). The second-generation hDPSCs were obtained and divided into three groups: normal group, sh-control group, and sh-circ104101 group. The results of qRT-PCR showed that there was no significant difference between the normal group and the sh-control group $(P>0.05)$. Compared with the sh-control group, the expression of the sh-circ104101 group decreased significantly $(P<0.01)$ (Figure 6(b)).

3.8. The Effect of hsa_circRNA_104101 on the Odontogenic Differentiation of hDPSCs. The staining of ALP decreased in the sh-circ104101 group compared with the normal and sh-control groups after induction for 7 days. Moreover, the contents of mineralized nodule formation of Alizarin red staining decreased in the sh-circ104101 group after odontogenic induction for 14 days (Figure 7). In addition, qRTPCR was used to detect the expression of mineralization- 


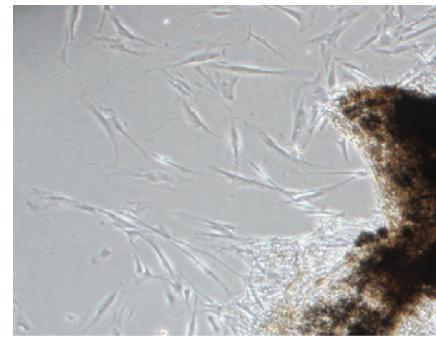

(a)

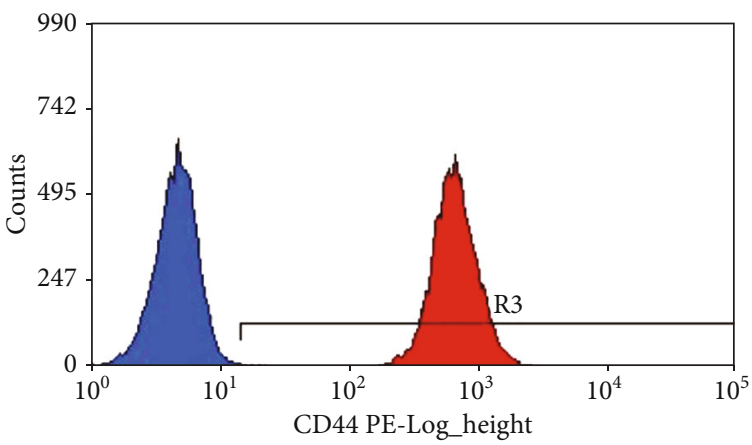

(c)

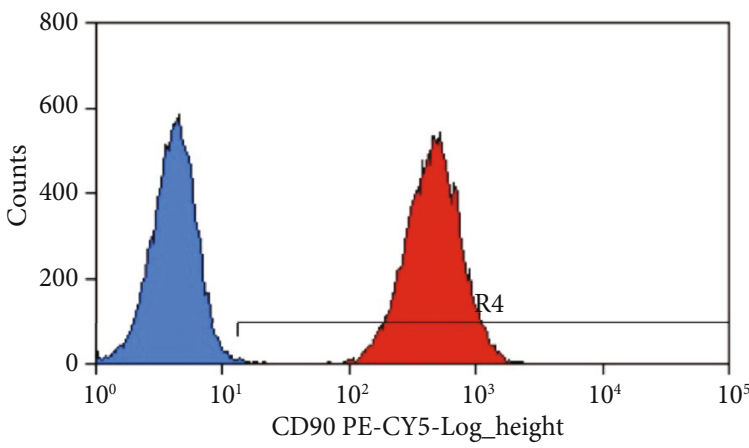

(e)

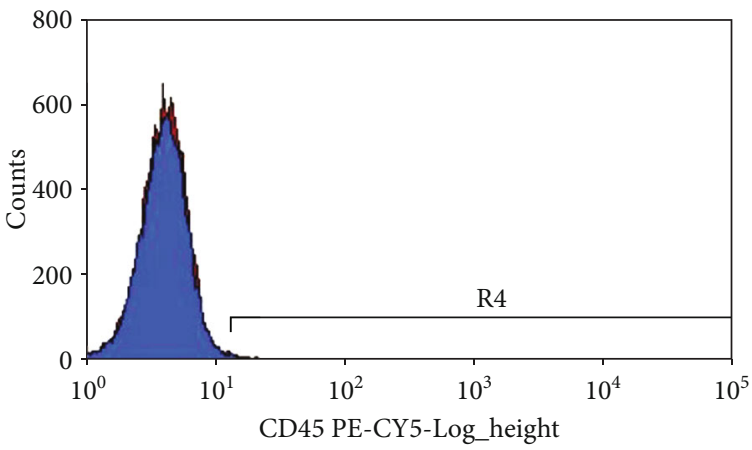

(g)

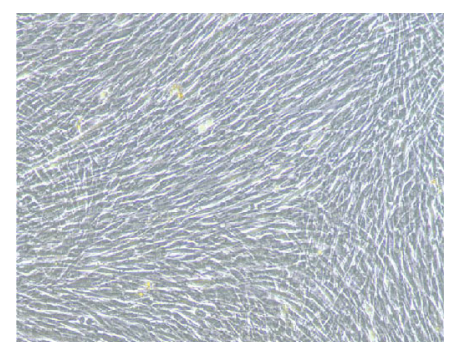

(b)

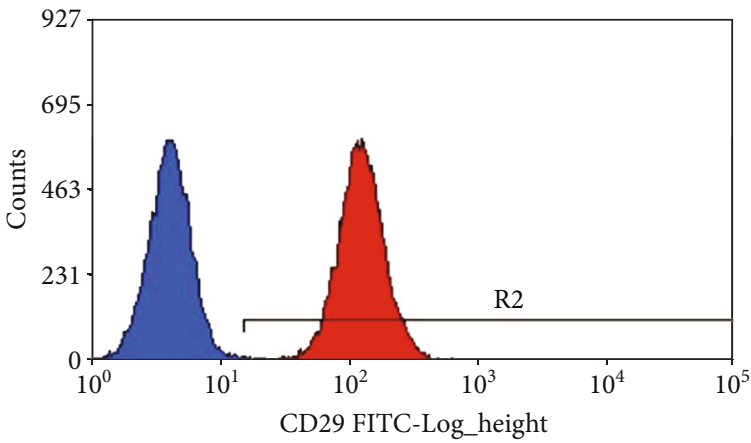

(d)

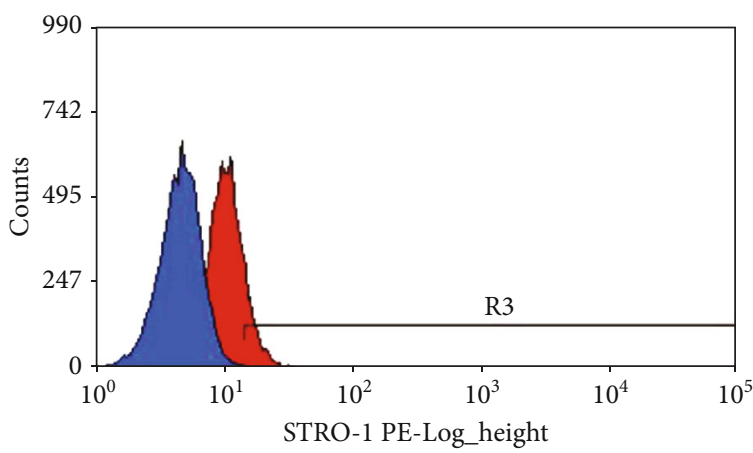

(f)

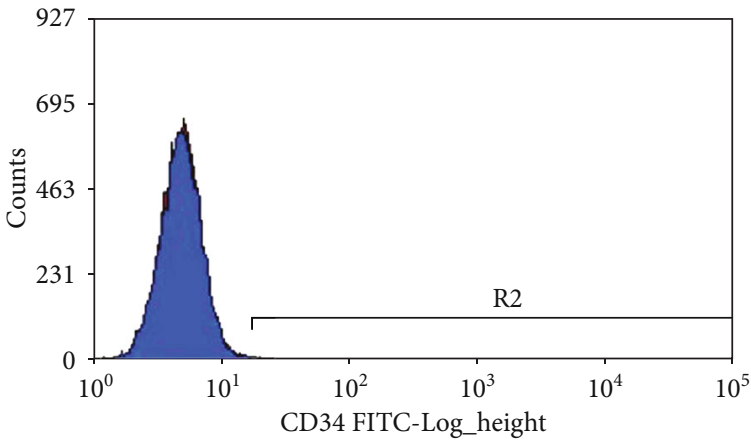

(h)

Figure 1: Continued. 


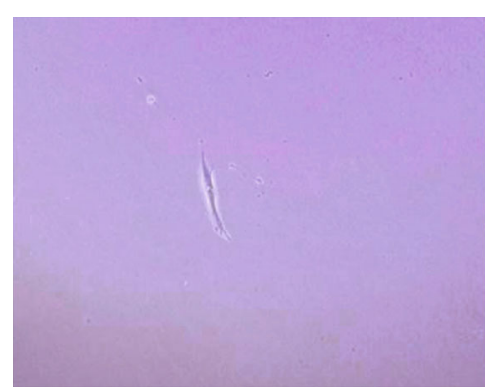

(i)

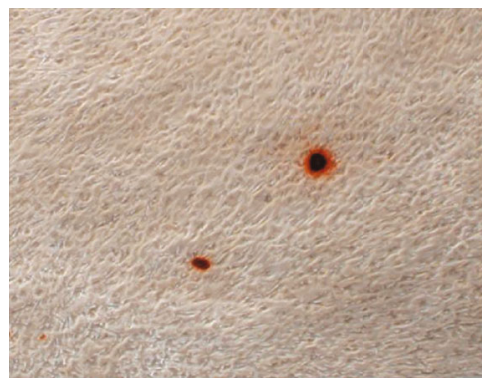

(k)

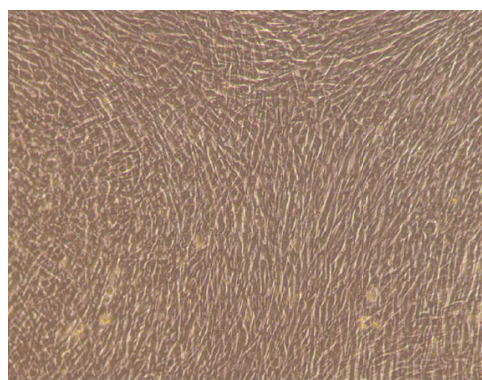

(m)

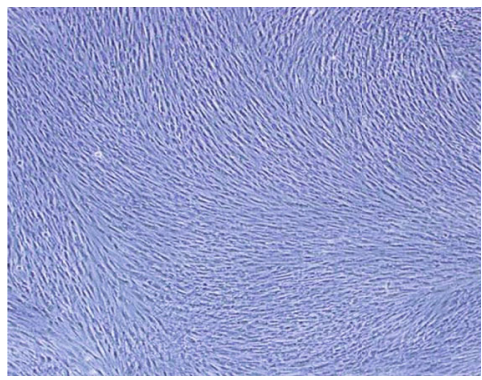

(o)

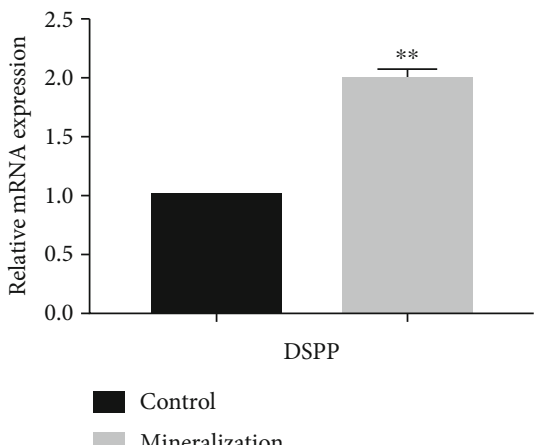

(q)

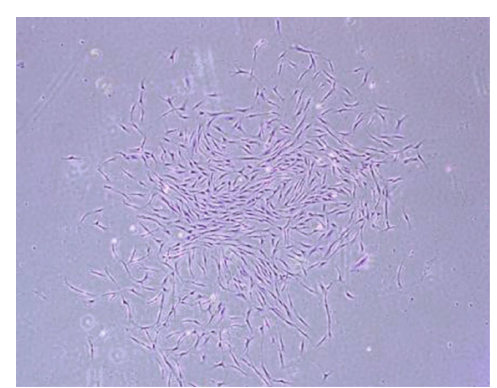

(j)

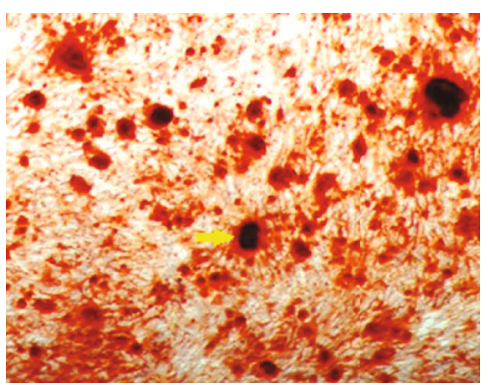

(1)

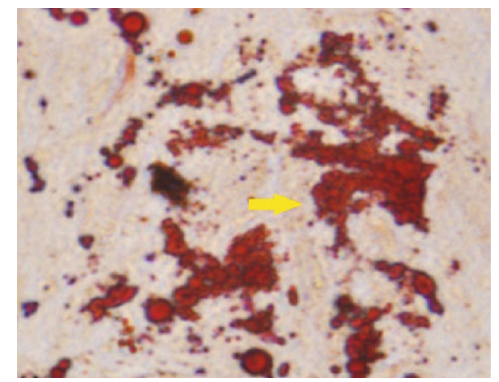

(n)

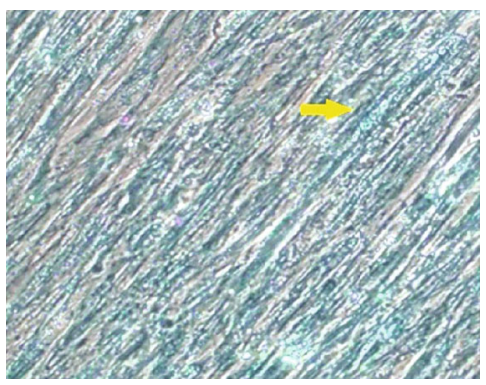

(p)

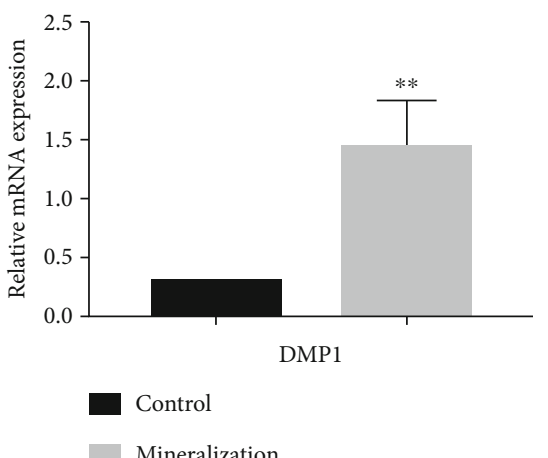

(r)

Figure 1: Continued. 


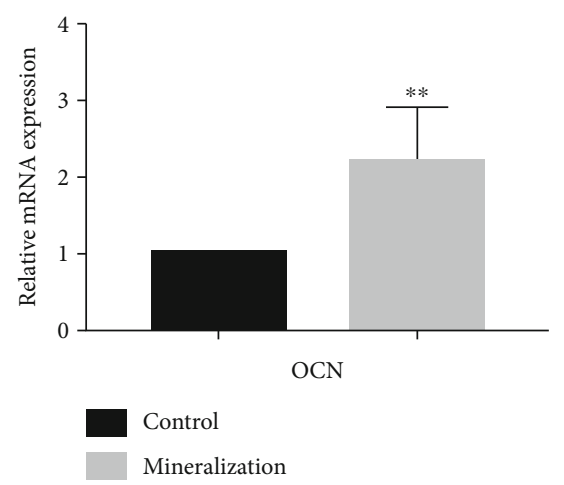

(s)

FIGURE 1: Identification and multiple differentiation potential of hDPSCs. (a) hDPSCs were separated from dental pulp. (b) hDPSCs were subcultured with normal media at third passage. (c-h) hDPSCs were positive for Stro-1 (18.39\%), CD44 (99.99\%), CD29 (97.96\%), and CD90 (99.99\%) and negative for CD45 (0.46\%) and CD34 (0.35\%). (i, j) A single cell was obtained, and single cell-derived colonies were obtained after culturing for 14 days. $(k, 1)$ Noninduced group and induced group of odontogenic differentiation. Mineral nodes formed in the induced group were as indicated by the yellow arrow. $(\mathrm{m}, \mathrm{n})$ Noninduced group and induced group of adipogenic differentiation. Lipid droplets formed in the induced group were indicated by the yellow arrow. (o, p) Noninduced group and induced group of chondrogenic differentiation. Expressed glycosaminoglycans formed in the induced group were indicated by the yellow arrow. (q-s) In the induced group, upregulated expressions of odontogenic differentiation-related genes DSPP, DMPI, and OCN by qRT-PCR were shown, compared with the noninduced group. All samples were performed in triplicate. The data are represented as means \pm SD. ${ }^{*} P<0.05,{ }^{* *} P<0.01$.

related factors in the normal group, the sh-control group, and the sh-circ104101 group after odontogenic induction for 7 and 14 days, respectively. The mRNA expressions of DSPP, DMP1, ALP, and OCN were downregulated after hsa_circRNA_104101 knockdown in hDPSCs (Figure 8). Western blot results showed that the protein levels of DSPP, DMP1, ALP, and OCN were also decreased in the sh-circ104101 group (Figure 9).

\section{Discussion}

circRNA molecules are more structurally stable than linear RNA [19]. In addition, circRNAs are widely distributed and have a high evolutionary conservation and tissue expression specificity [20]. In some cases, the circRNA has an abundance of more than 10 times compared to the relative linear mRNA. Therefore, circRNAs are considered to be the ideal disease diagnostic marker and therapeutic target and have a great potential clinical value $[21,22]$. In recent years, many investigators demonstrated that long noncoding RNA (lncRNA), miRNA, and other ncRNAs are involved in the hDPSC odontogenic differentiation. However, the role of circRNA in this process was poorly understood. In our study, the expression of 12929 circRNAs was detected during hDPSC odontogenic differentiation using microarray analysis. For about $1 \%$ of them (187), we found a significantly different expression in the differentiated state compared to the undifferentiated state (fold change $>1.5$ or $<-1.5$ ). Of the 187 differently expressed circRNAs, 43 were upregulated and 144 downregulated. The most significantly upregulated circRNAs are transcribed from exons (19-fold change). This suggests that circRNA may play a significant role in the odontogenic differentiation of hDPSCs.
To further understand the potential regulatory role of the differentially expressed circRNAs, we performed KEGG pathway and GO analysis to evaluate which cellular functions involved the circRNA target genes. In the GO analysis, we found that the target genes were correlated with many important biological processes, including odontogenic differentiation. In particular, the more represented functional classes were the following: cellular communication and signal transduction. By performing the KEGG analysis, we predicted that the target genes were correlated with the Wnt pathway, TGF- $\beta$ pathway, and other signaling pathways regulating stem cell pluripotency. This is in agreement with a previous study that reported that the Wnt signaling pathway has an important role in odontoblast biology [36]. Moreover, it is already known that ncRNAs can inhibit the odontogenetic differentiation of hDPSCs through Wnt signaling pathway inhibition [32]. Additionally, the TGF- $\beta$ pathway is essential in the repair of pulp injury: when dentin is damaged, the dentin matrix releases TGF- $\beta 1$, which can induce dentin cells to regenerate to repair the tissue [37]. Thus, our results suggest that the differentially expressed circRNAs detected in this study regulate odontogenic differentiation through these two pathways.

It has been shown that circRNA plays an important role in the osteogenic differentiation of a variety of stem cells. However, the research on the role of circRNA in the odontogenic differentiation of hDPSCs only focuses on the analysis of high-throughput sequencing results and bioinformatics [38]. Therefore, we selected one predicted circRNA based on our results for subsequent functional experiments. In order to study the effect of hsa_circRNA_104101 on the odontogenic differentiation of hDPSCs, we carried out the hsa_circRNA 104101 RNA interference silencing experiment. RNA interference can specifically knock down the target gene expression, which is the most widely used technology to study the 


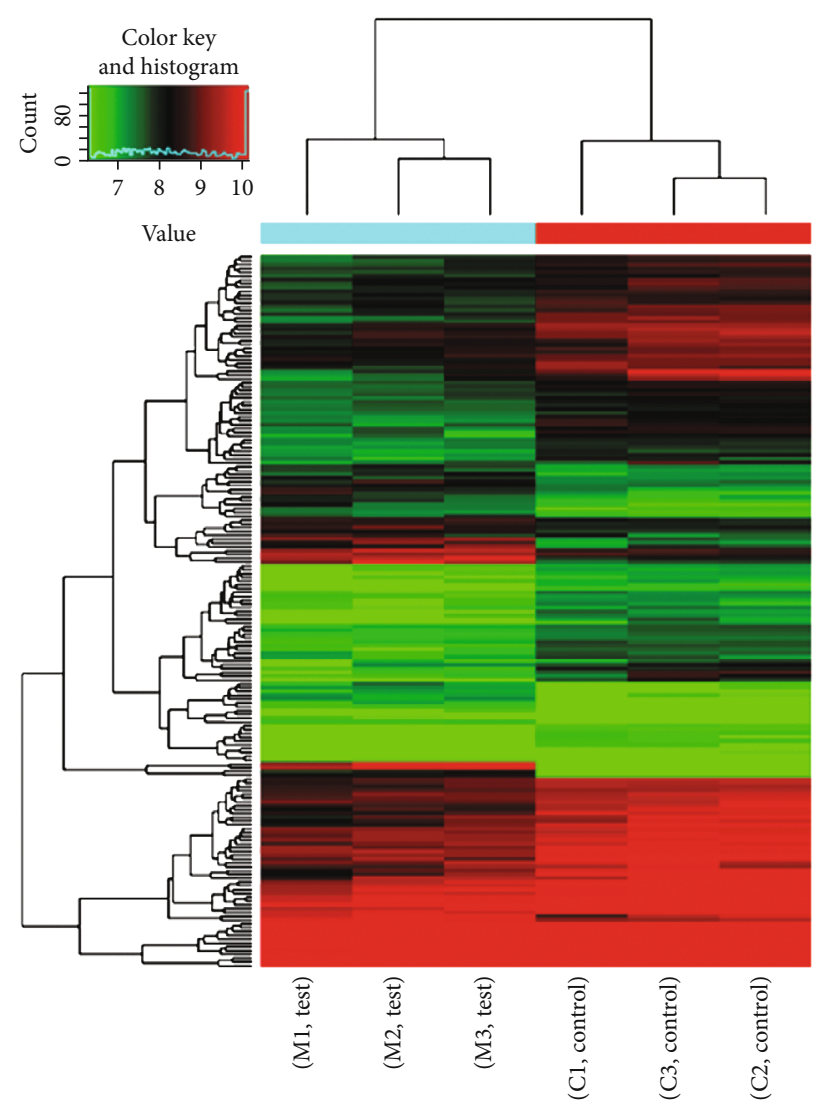

(a)
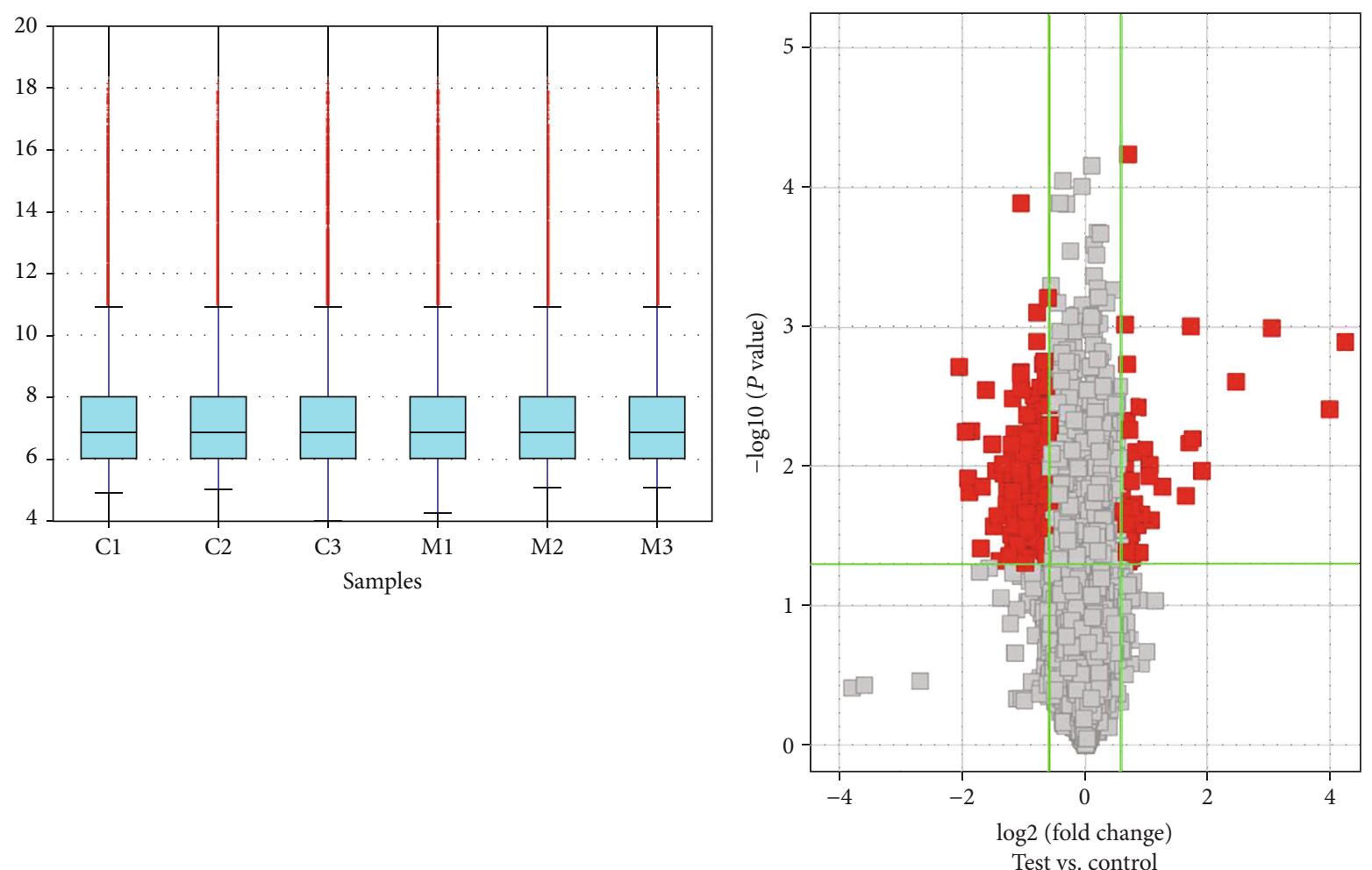

(b)

(c)

Figure 2: Continued. 


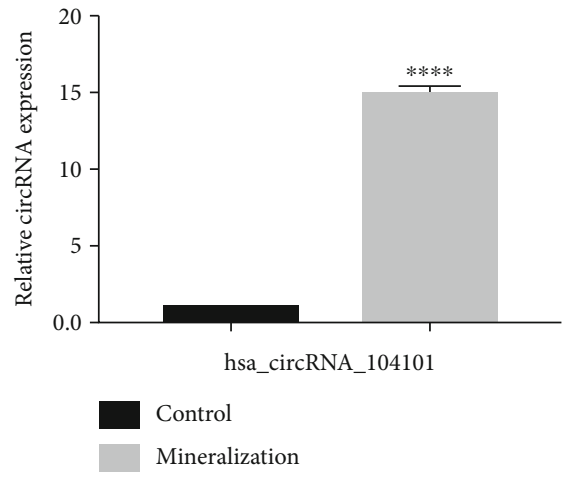

(d)

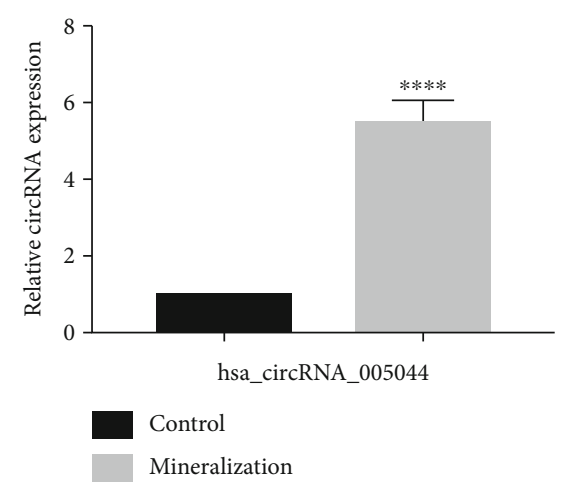

(g)

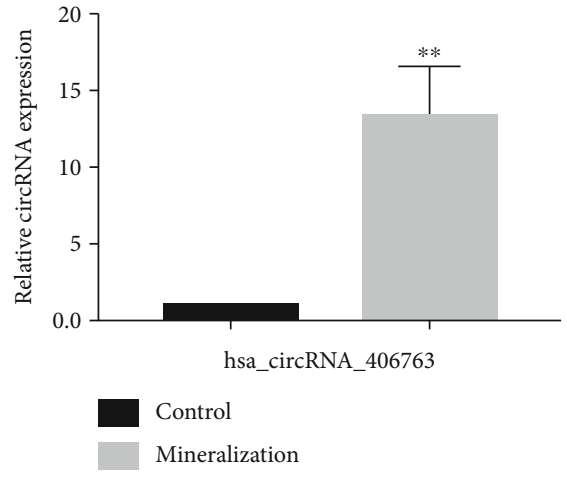

(e)

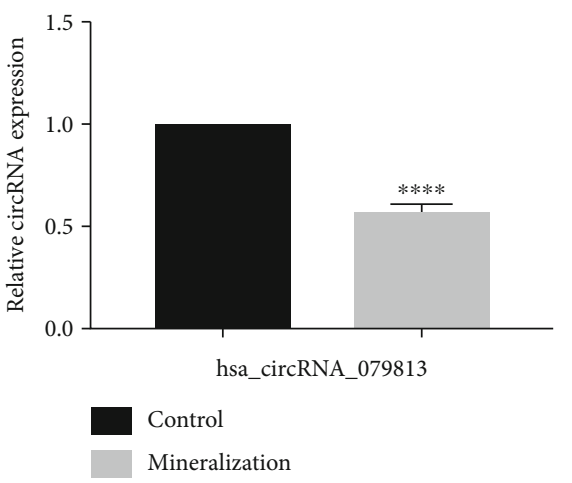

(h)

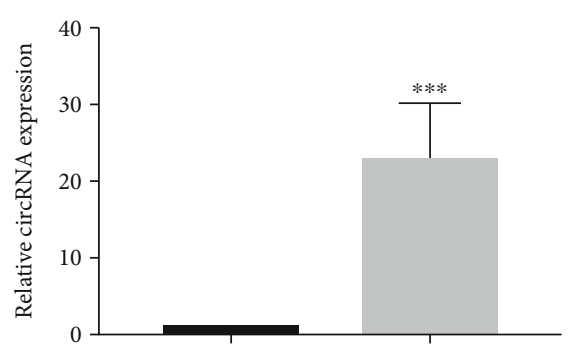

hsa_circRNA_002161

Control

Mineralization

(f)

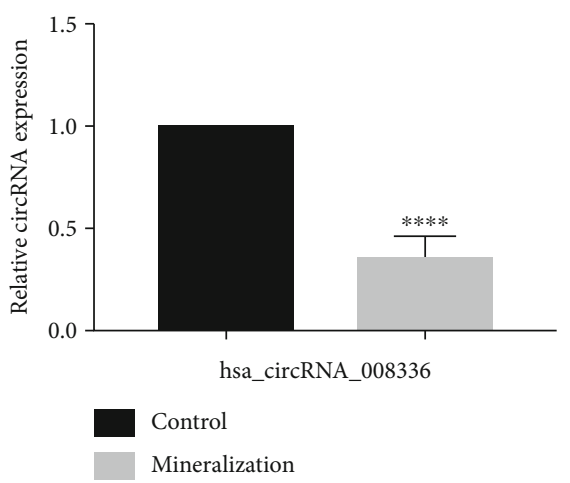

(i)

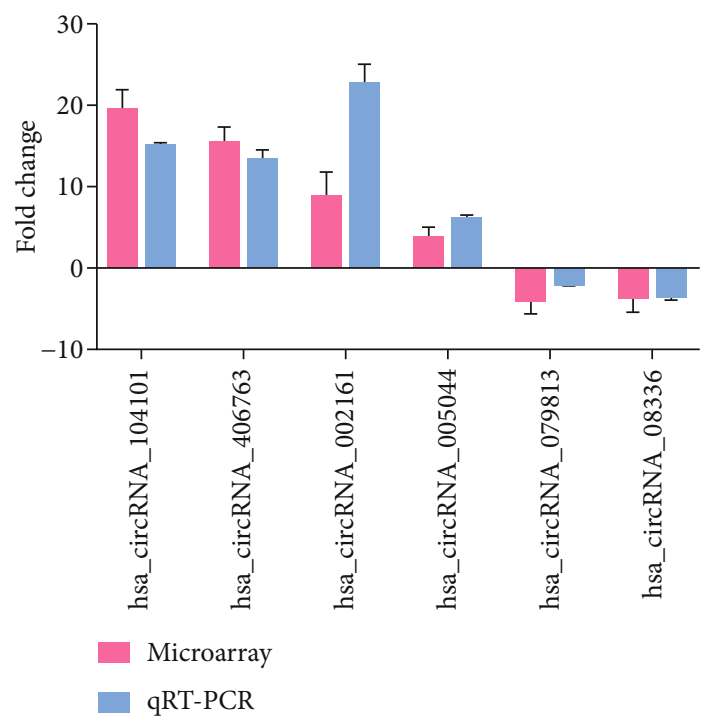

(j)

FIGURE 2: Differentially expressed circRNAs between different groups, differentially expressed circRNAs validated by qRT-PCR. (a) Hierarchical cluster analysis of circRNAs in the induced and noninduced groups by gene chip. The parts of red and green, respectively, mean the upregulation and downregulation of circRNA. (b) Box plot indicated that the distribution of the intensities among all samples was nearly the same. (c) Red font in the volcano plot denotes the differentially expressed circRNAs with statistical significance from hDPSCs between the induced and noninduced groups (fold change $>1.5$ or $<-1.5, P<0.05$ ). (d-g) circRNA hsa_circRNA_104101, hsa_ circRNA_406763, hsa_circRNA_002161, and hsa_circRNA_005044 were upregulated in the induced group in comparison with the noninduced group. $(\mathrm{h}, \mathrm{i})$ circRNAs hsa_circRNA_079813 and hsa_circRNA_008336 were downregulated. The data were represented as means \pm SD. ${ }^{*} P<0.05,{ }^{* *} P<0.01,{ }^{* *} P<0.001$, and ${ }^{* * * *} P<0.0001$. (j) Results of the comparison of microarray and qRT-PCR data for candidate circRNAs. 


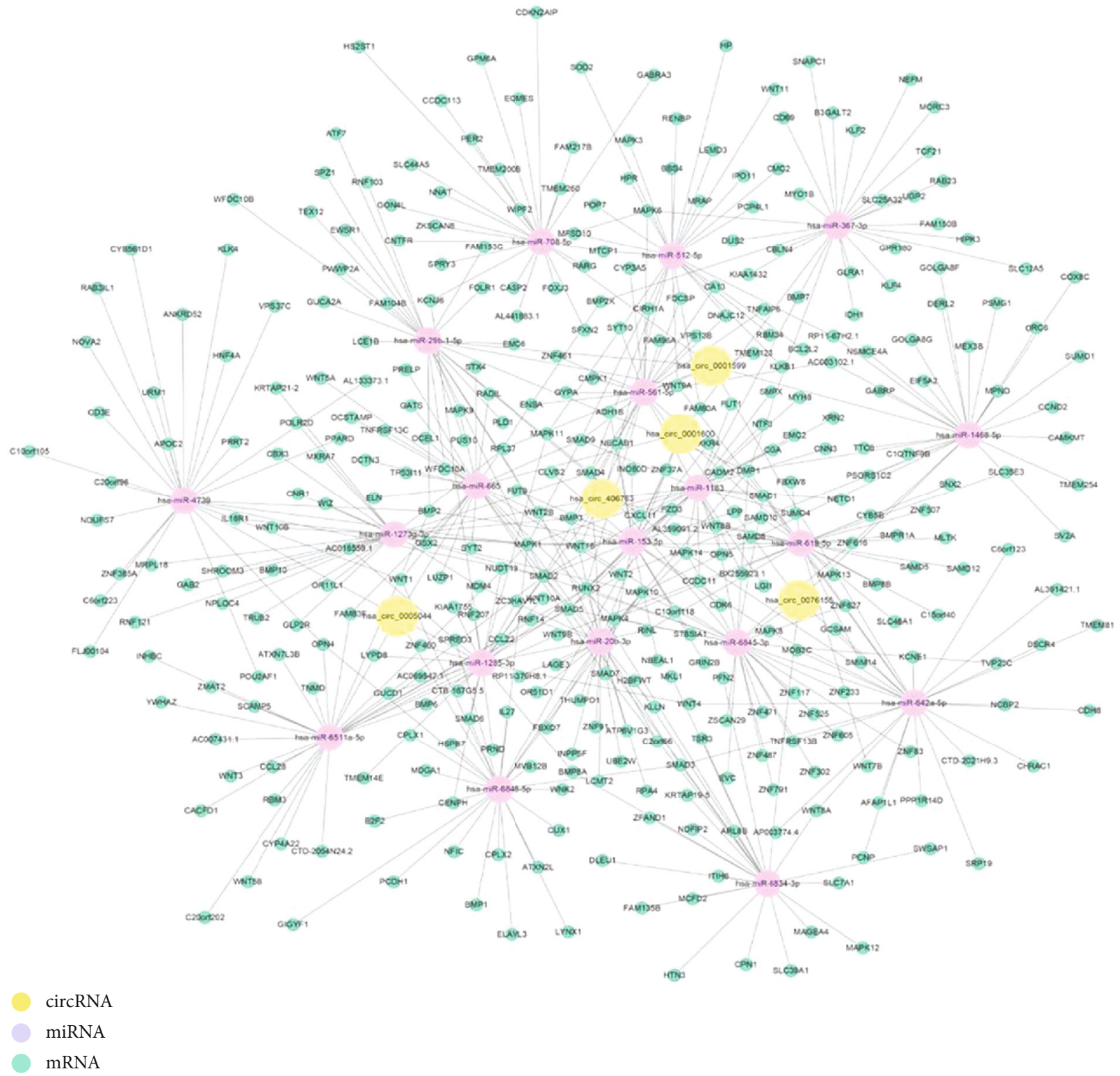

(a)

Figure 3: Continued. 


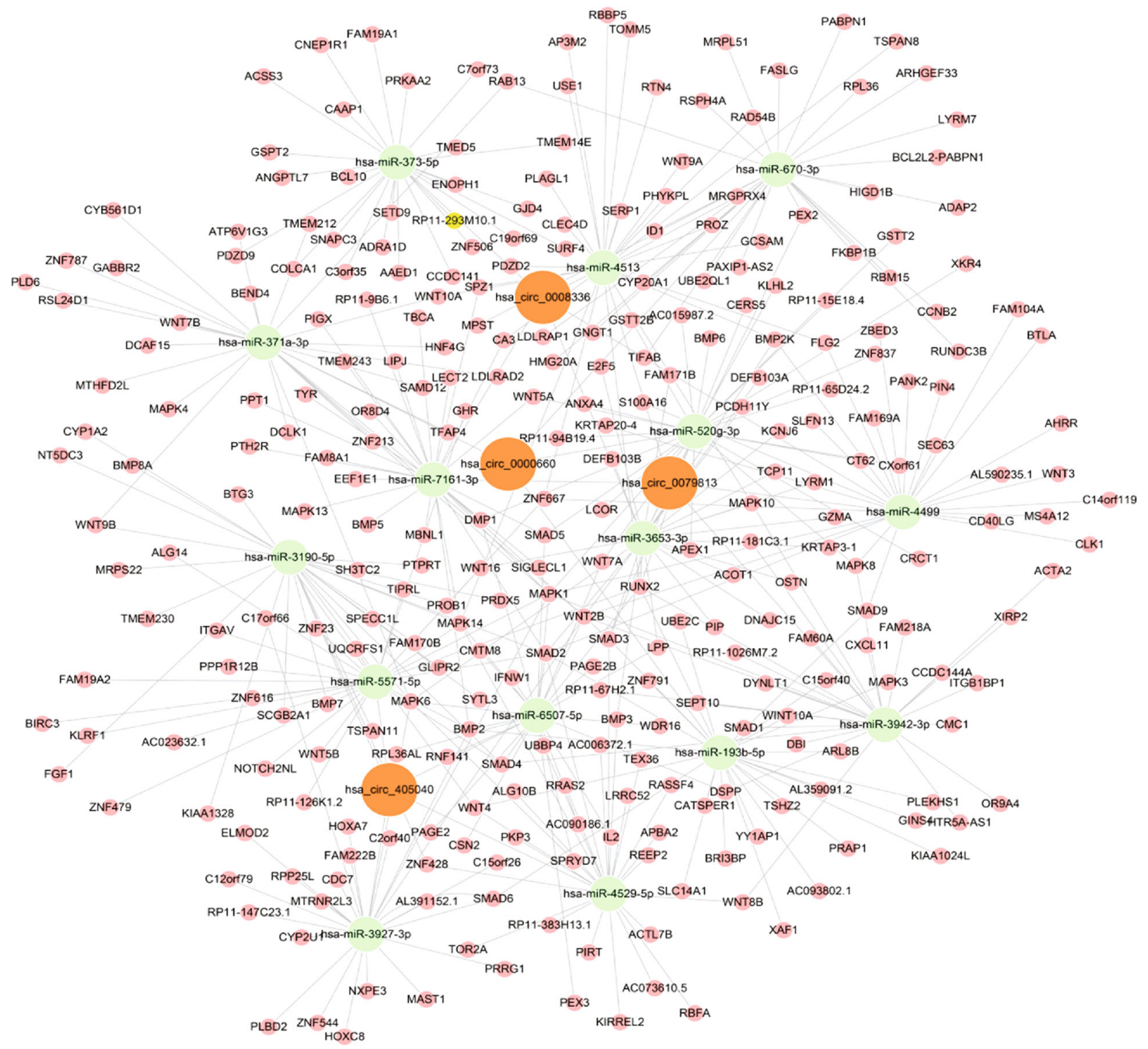

circRNA

miRNA

mRNA

(b)

FiguRE 3: circRNA-miRNA-mRNA network. Several most significantly different circRNAs were selected from the results of the gene chip, miRanda, and Targetscan software which were used to determine the circRNAs combined with miRNAs, and TargetScan, miRDB, CircBase, and CircBank were used to predict target mRNAs. (a) CeRNA networks of upregulated circRNAs-miRNAs-mRNAs, included 5 circRNAs, 19 miRNAs, and 363 mRNAs. Yellow circle represents circRNAs, purple circle represents miRNAs, green circle represents underlying target mRNAs, and the lines indicate interactions between circRNAs and miRNAs or miRNAs and mRNAs. (b) CeRNA networks of downregulated circRNAs-miRNAs-mRNAs, included 4 circRNAs, 16 miRNAs, and 258 mRNAs. Orange circle represents circRNAs, light green circle represents miRNAs, pink circle represents underlying target mRNAs, and the lines indicate interactions between circRNAs and miRNAs or miRNAs and mRNAs.

biological function of a gene. In our experiment, shcirc104101 was successfully synthesized and packaged into lentivirus, which successfully inhibited the expression of hsa_circRNA_104101. After 14 days of mineralization induction, the odontogenic differentiation-related genes and pro- teins were detected by $\mathrm{qRT}-\mathrm{PCR}$ and Western blot. It was found that the expression of DSPP, DMP1, ALP, and OCN decreased in varying degrees. DSPP is an important noncollagenous protein in the dentin matrix, which is mainly expressed in odontoblasts, and is closely related to tooth 


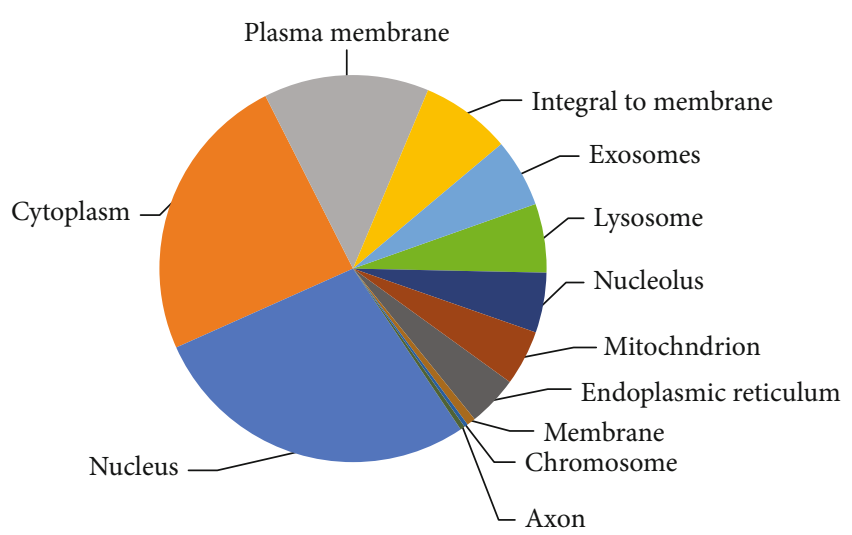

(a)

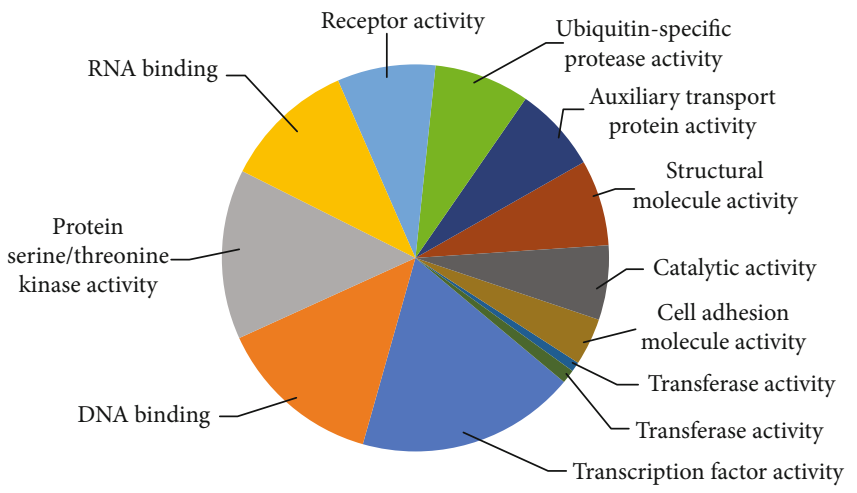

(c)

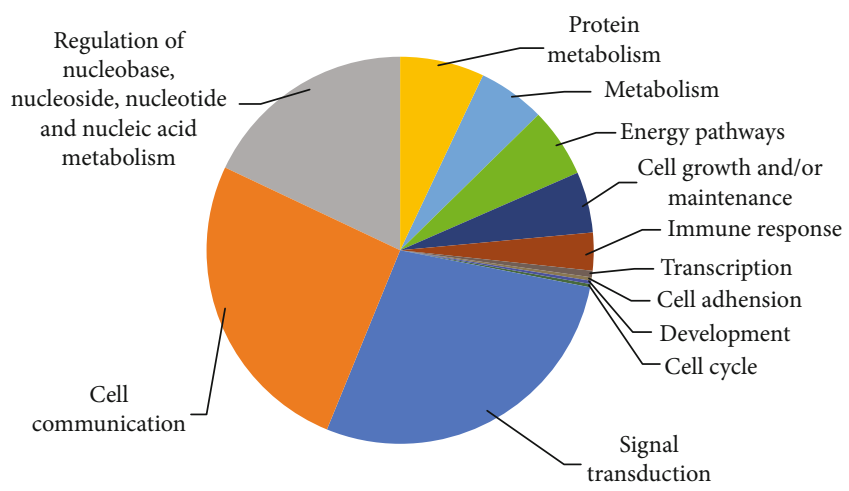

(e)

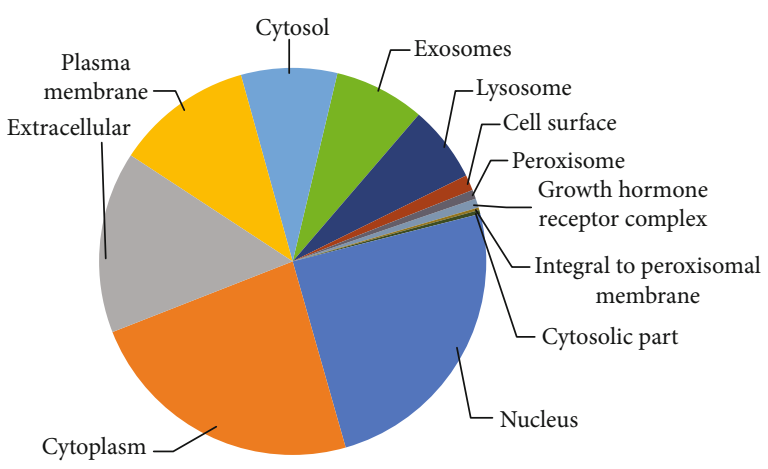

(b)

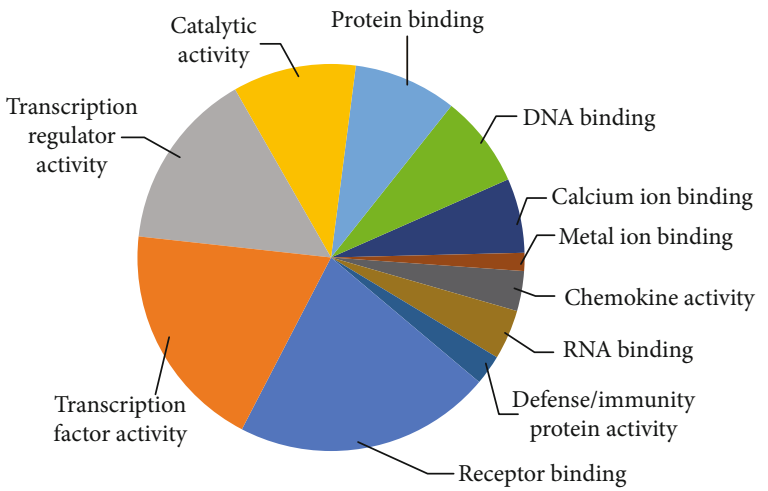

(d)

Regulation of

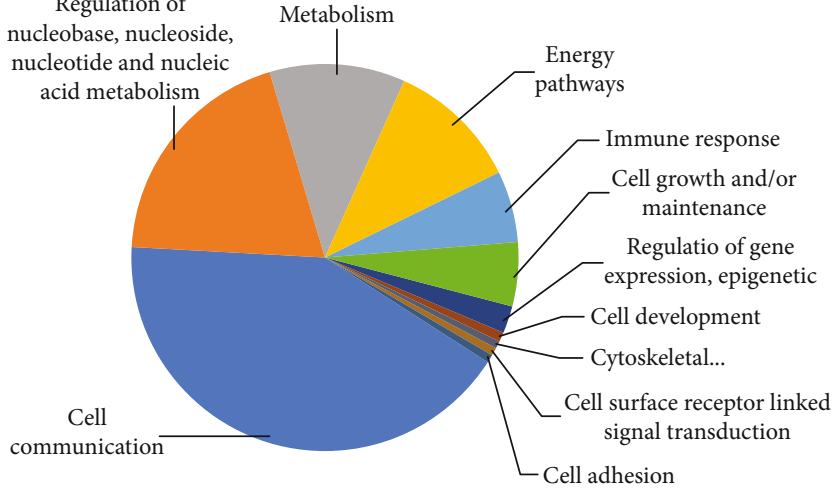

(f)

Figure 4: Continued. 


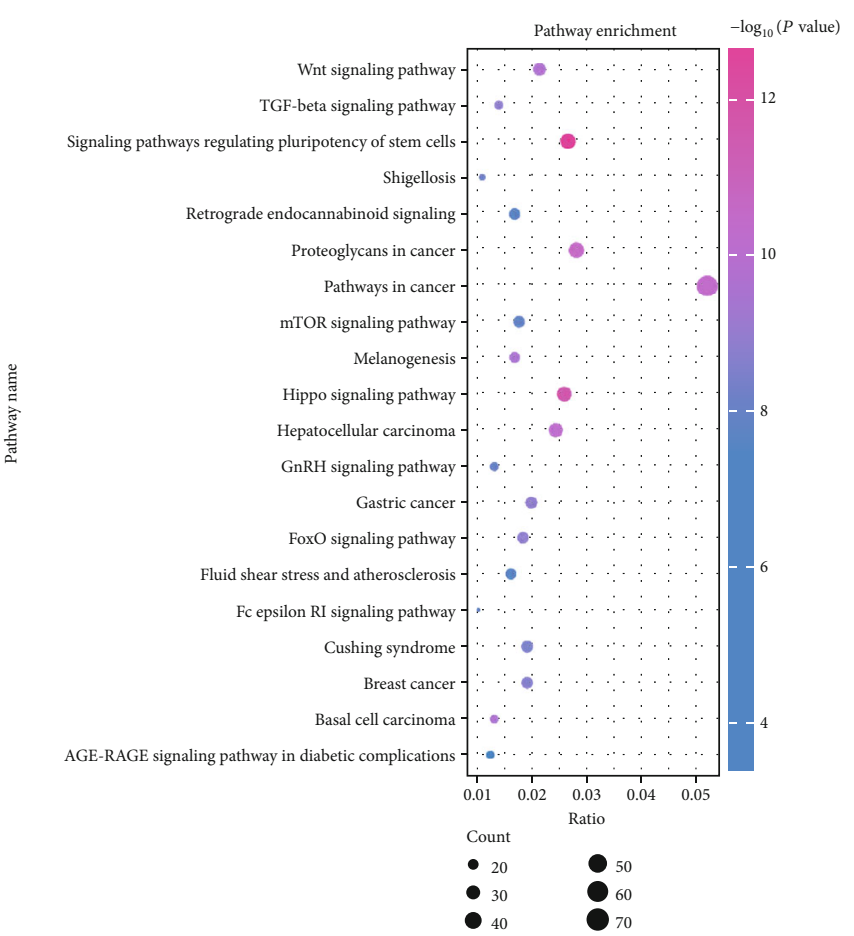

(g)

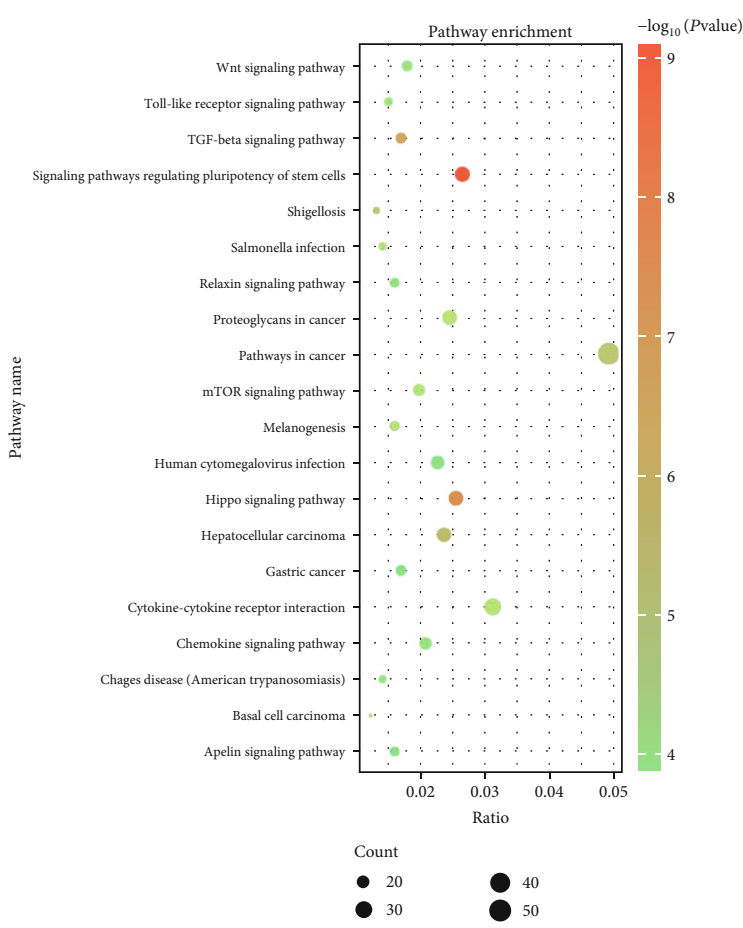

(h)

FIGURE 4: GO and KEGG pathway analysis of noninduced and induced hDPSCs. GO analysis of 1345 upregulated differentially expressed mRNAs and 1058 differentially expressed downregulated mRNAs, respectively. (a) GO cellular component classification of upregulated mRNAs. (b) GO cellular component classification of downregulated mRNAs. (c) GO molecular function classification of upregulated mRNAs. (d) GO molecular function classification of downregulated mRNAs. (e) GO biological process classification of upregulated mRNAs. (f) GO biological process classification of downregulated mRNAs. KEGG pathway enrichment. The $X$-axis represents the ratio of enriched differentiated genes to all genes enriched for a particular pathway term. Spot size represents the number of significantly differentiated genes; larger spots indicate more differentiated genes; the color of circle indicates the $P$ value including the signaling pathways regulating pluripotency of stem cells, the Wnt pathway, and the TGF- $\beta$ pathway. (g) KEGG pathway analysis of upregulated mRNAs. (h) KEGG pathway analysis of downregulated mRNAs.

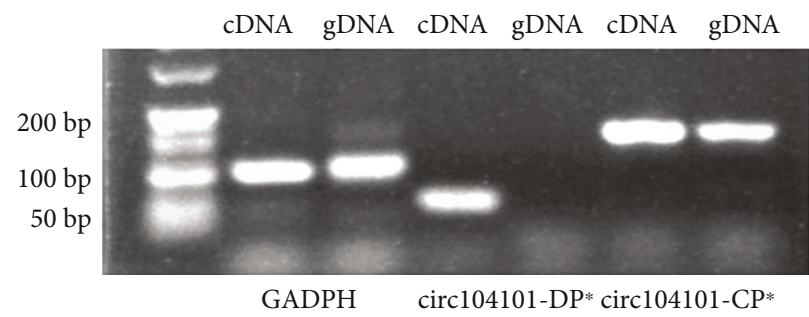

(a)

\begin{tabular}{llllll}
\hline 50 & 60 & 70 & 80 & 90 & 100
\end{tabular}

G G CAC C T T CA T CA G T A G T CA T T G T C T T T TA A G T G A G A A C T T T C T A C T G T T G T C

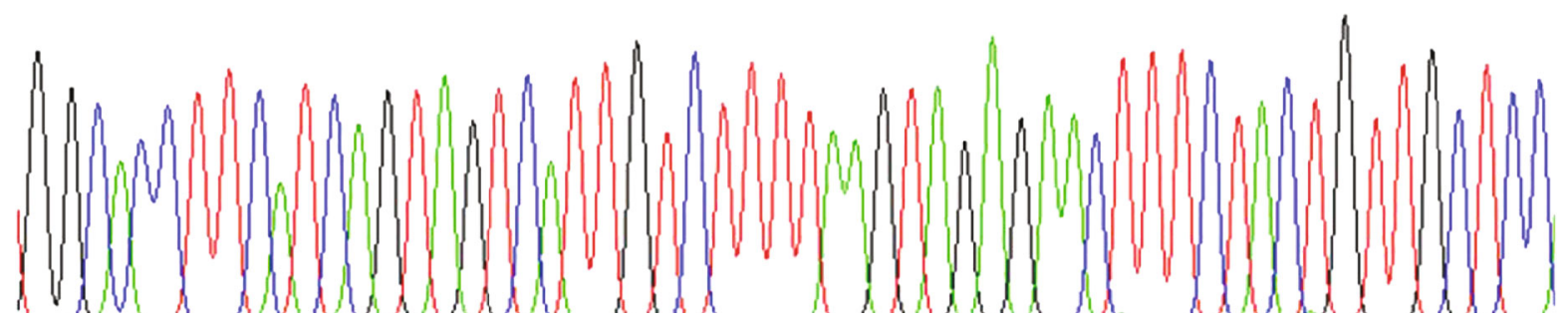

(b)

FiguRE 5: Identification of hsa_circRNA_104101. (a) Agarose gel electrophoresis experiment. *: DP: divergent primer; CP: convergent primer. (b) Sanger sequencing. 


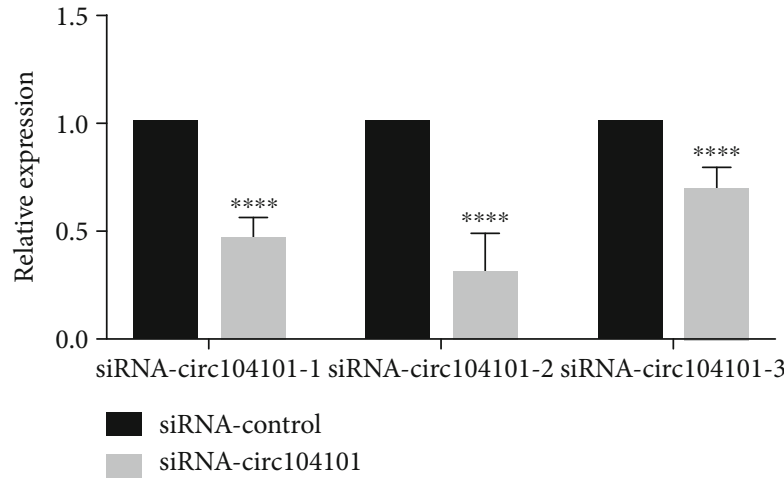

(a)

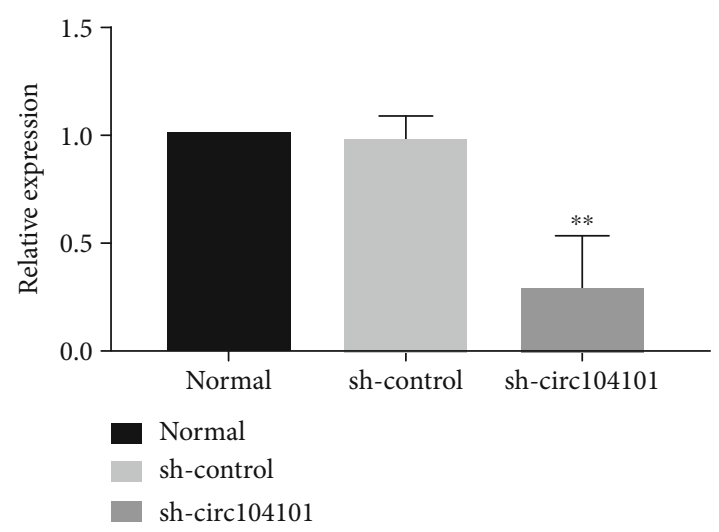

(b)

FIGURE 6: Construction of hsa_circRNA_104101 shRNA lentivirus and cell infection. (a) siRNA cell transfection (the data were represented as means \pm SD for each group: $\left.{ }^{* * *} P<0.001,{ }^{* * * *} P<0.0001\right)$. (b) Knockdown of hsa_circRNA_104101 after lentivirus transfection was confirmed by qRT-PCR (the data were represented as means \pm SD for each group, ${ }^{* *} P<0.01$ ).

development and biomineralization. DMP1 is a noncollagenous extracellular matrix protein of dentin, which plays an important role in the odontogenic differentiation of undifferentiated mesenchymal cells [23]. ALP is considered to be an important marker in the early stage of osteogenesis, which promotes calcification in the formation of hard tissue. OCN is one of the differentiation markers from osteoblasts to mineralization stage. At the same time, Alizarin red staining showed that the formation of calcium nodules was reduced. The above results indicated that the silencing of hsa_circRNA_104101 could inhibit the odontogenic differentiation of hDPSCs, which provided the experimental basis for the follow-up mechanism study, and also provided a reference for the role of other circRNAs in the process of odontogenic differentiation of hDPSCs.

Several gene chip data results available from different databases were used for constructing a circRNA-miRNAmRNA network. Multiple miRNAs and mRNAs can be regulated by a singular circRNA through different mechanisms, revealing a complex interaction between these molecules. Interestingly, some of the miRNAs detected in this network have been already demonstrated to regulate differentiation processes. For instance, it has been reported that, in peripheral blood monocytes, miR-708-5p can negatively regulate the expression level of factors involved in bone metabolism such as AKT1, AKT2, PARP1 FKBP5, and MP2K3, resulting in an osteoporotic phenotype [39]. Moreover, the lncRNA HIF1A-AS2 can competitively bind miR-665 and thus positively regulates IL6, activating the PI3K/Akt signaling pathway, which promotes osteogenic differentiation of adiposederived stem cells [40]. Overexpression of miR-665 was found to decrease the recruitment of the negative regulators KAT6A to DSPP and DMP1 gene promoters during dentinogenesis [41]. Elsafadi et al. reported that miR-4739 overexpression could inhibit the osteogenic differentiation of human bone marrow stromal cells [42]. In our ceRNA network, we predicted that the odontogenic differentiationrelated genes DSPP and DMP1 might be correlated to hasmiR-708-5p and has-miR-642a-5p. In turn, these miRNAs may be correlated to the differentially expressed circRNAs during hDPSC odontogenic differentiation, although these potential novel regulatory pathways should be experimentally confirmed in future studies.

It is generally accepted that stem cells have various differentiation potential, which is regulated by the presence of different signal molecules and bioactive materials. In the context of odontogenic differentiation, stem cells differentiate into pulp, dentin, and other cell types [43]. Pulp revascularization has been widely used for the treatment of pulp necrosis. However, it was extensively reported that the tissues in the root canals consist of cementum-like, bone-like, and periodontal ligament-like tissues [44, 45]. Induced repair of dental pulp is probably different from the real physical regeneration $[46,47]$.

Therefore, the follow-up experiments of our study were mainly focused on whether hsa_circRNA_104101 can regulate the mineralization process as a ceRNA function. We first intend to further utilize fluorescence in situ hybridization to locate hsa_circRNA_104101, then identify miRNA molecules interacting with circRNA by RNA pull-down experiment, and detect specific binding sites by a dual luciferase reporter system to further analyze its mechanism.

\section{Conclusion}

In summary, we found that 187 circRNAs were differentially expressed in hDPSCs during odontogenic differentiation. Moreover, we performed a bioinformatic analysis to detect pathways linked to odontogenic differentiation through circRNAs. We predicted that the Wnt and TGF- $\beta$ signaling pathway, as well as the other pathways associated with odontogenic differentiation, are correlated to the differentially expressed circRNAs. We constructed a circRNA-miRNAmRNA network, which may provide a new theoretical foundation for the study of pulp regeneration. Our results unveil the potential role of circRNA in odontogenic differentiation. In addition, our study indicated that hsa_circRNA_104101 was involved in the odontogenic differentiation of hDPSCs. circRNAs could be novel therapeutic targets for pulp regeneration. 


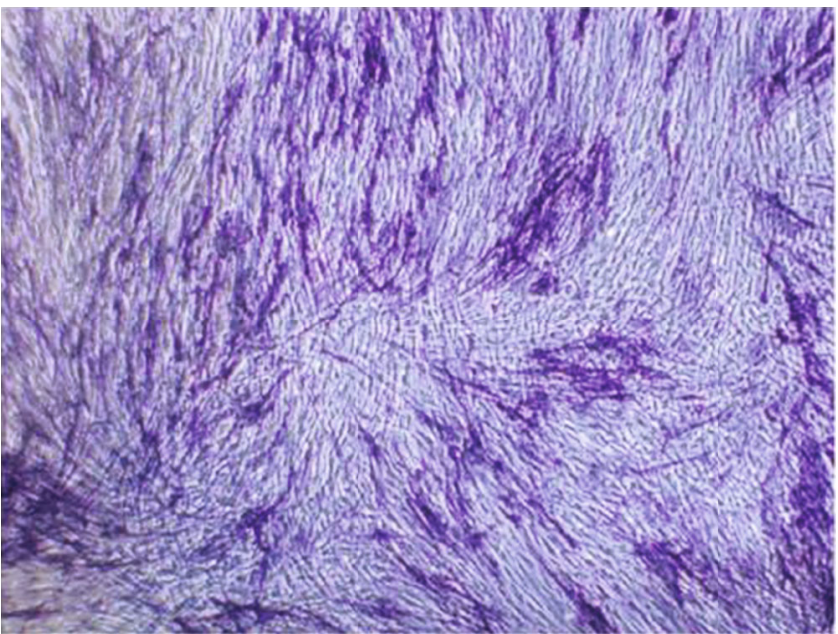

(a)

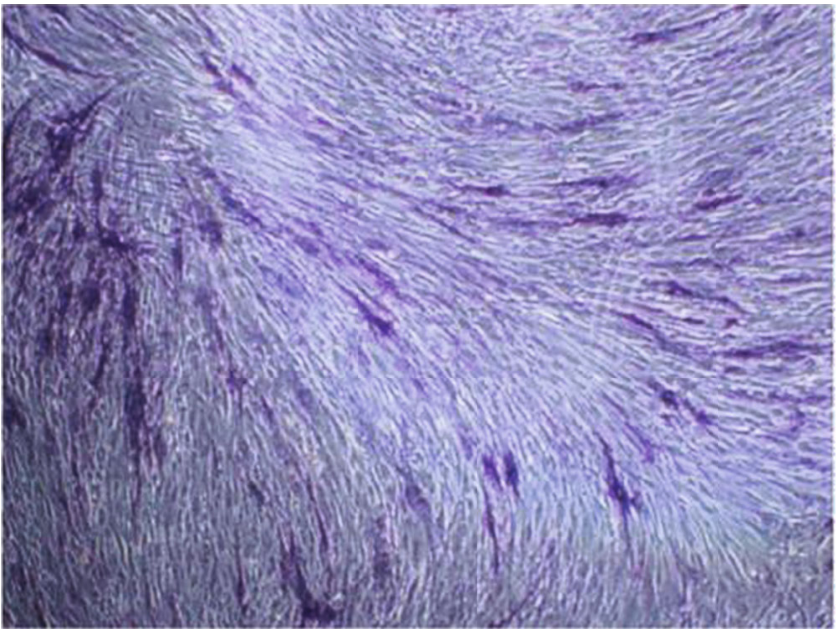

(c)

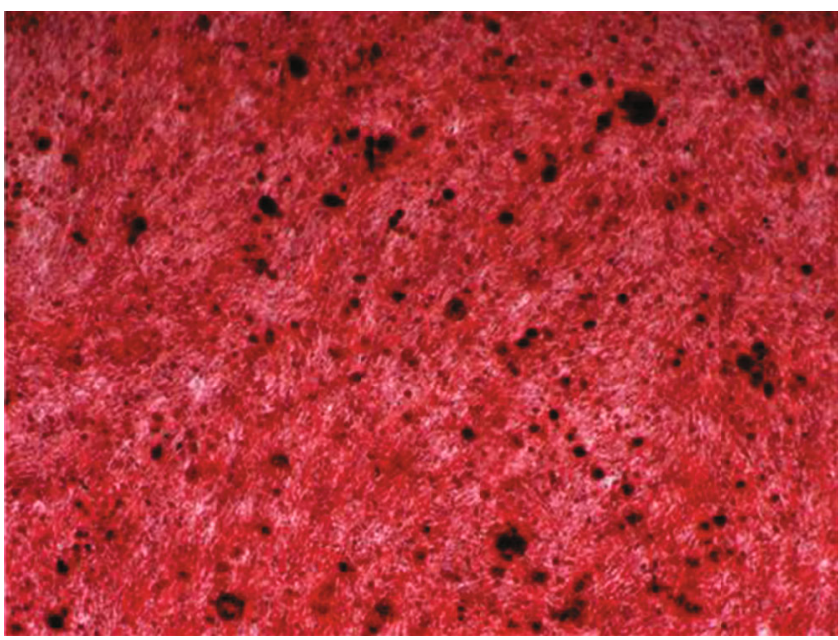

(e)

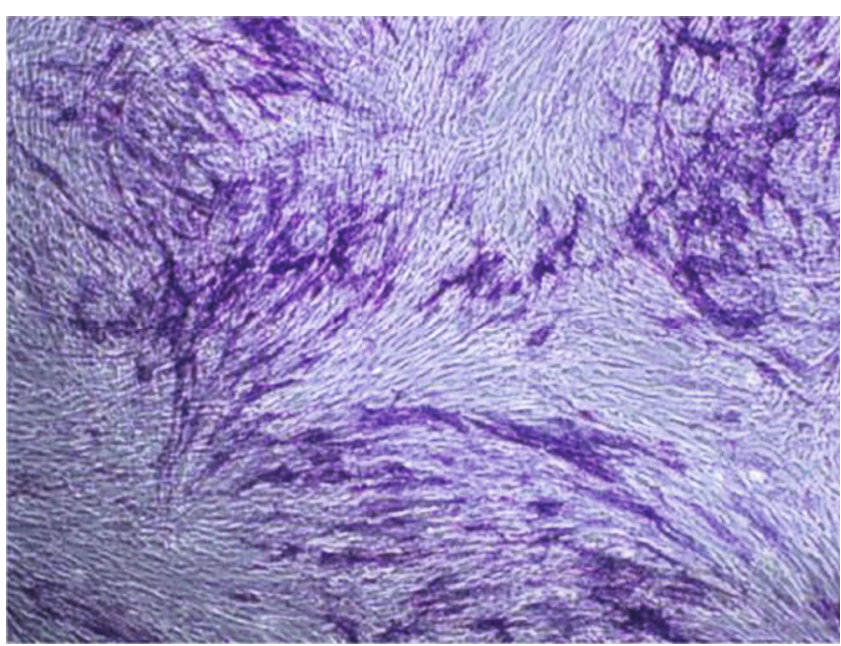

(b)

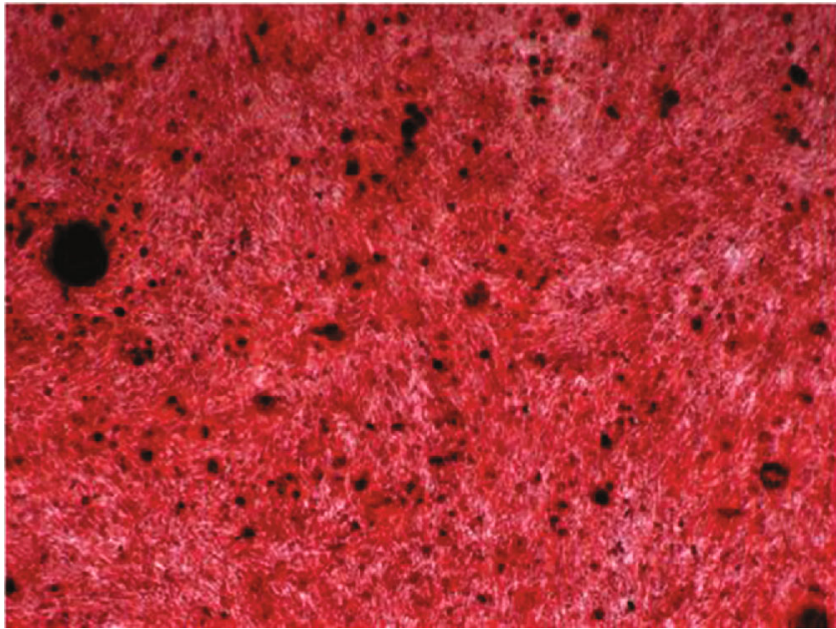

(d)

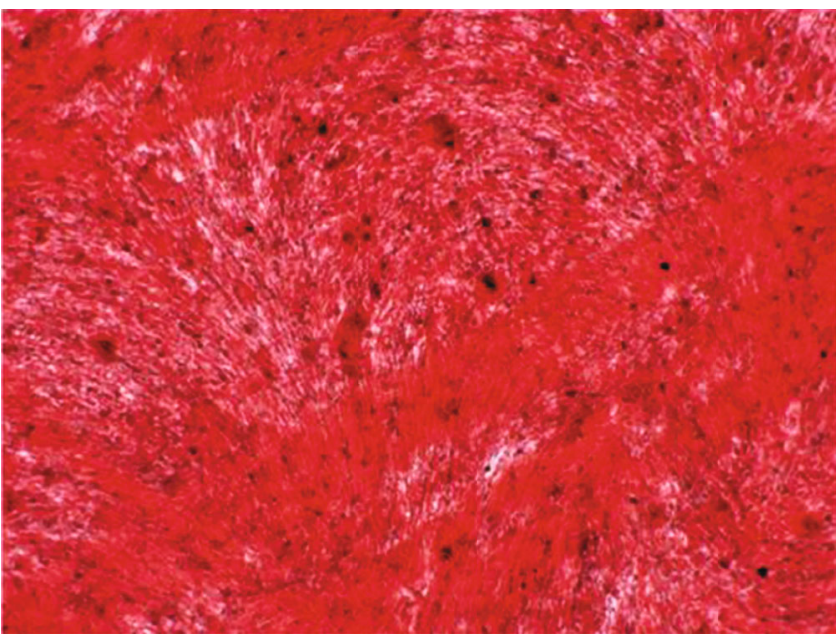

(f)

FIgURE 7: ALP activity examination and Alizarin red staining. (a) ALP staining in normal group. (b) ALP staining in sh-control group. (c) ALP staining in sh-circRNA group. (d) ARS staining in normal group. (e) ARS staining in sh-control group. (f) ARS staining in shcircRNA group. 


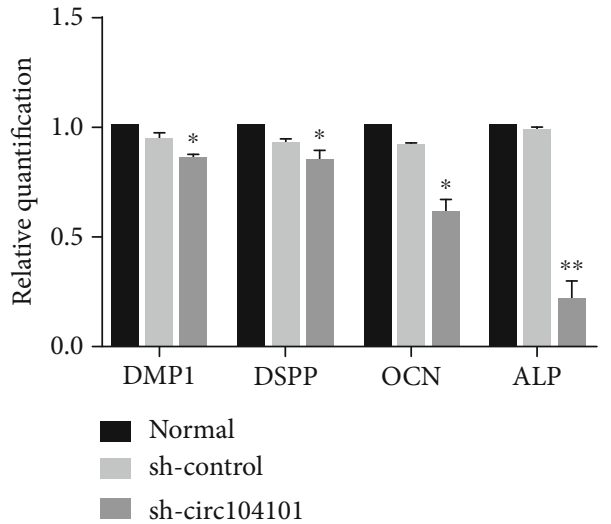

(a)

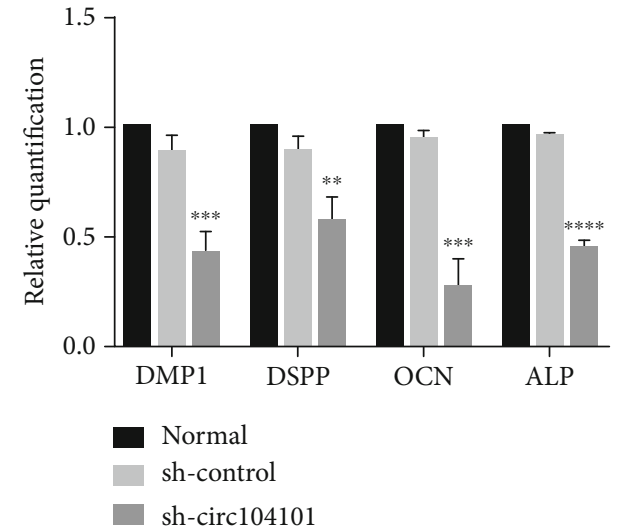

(b)

FIGURE 8: Expression of mineralization-related mRNA after hDPSC transfection. (a) The expression level of DMP1, DSPP, OCN, and ALP decreased in the sh-circ104101 group at 7 days after odontogenic differentiation using qRT-PCR. (b) The expression level of DMP1, DSPP, OCN, and ALP decreased in the sh-circ104101 group at 14 days after odontogenic differentiation using qRT-PCR (the data were represented as means \pm SD for each group: ${ }^{*} P<0.05,{ }^{* *} P<0.01,{ }^{* * *} P<0.001$, and $\left.{ }^{* * * *} P<0.0001\right)$.

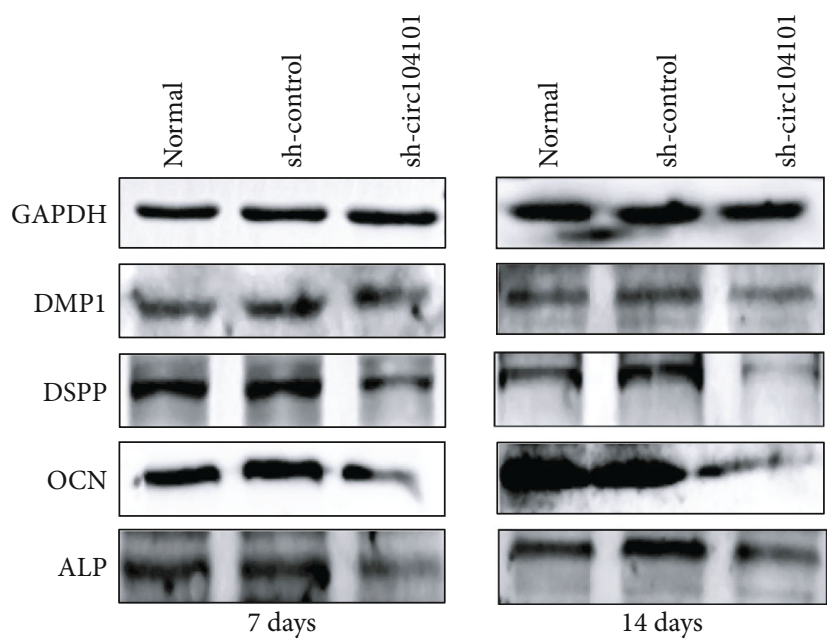

(a)

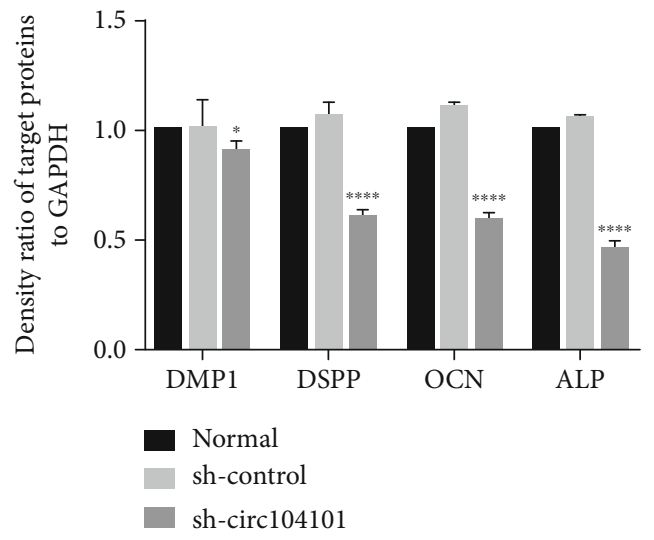

(b)

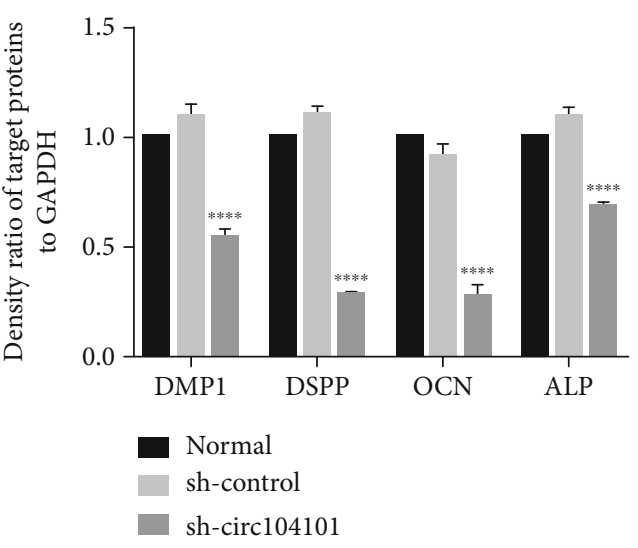

(c)

FIGURE 9: Expression of mineralization-related proteins after hDPSC transfection. (a) Western blot analysis shows the expression level of DMP1, DSPP, OCN, and ALP decreased in the sh-circ104101 group after odontogenic differentiation for 7 and 14 days. GAPDH was used as an internal control. The data were represented as means $\pm \mathrm{SD}$ for each group: ${ }^{*} P<0.05,{ }^{* * * *} P<0.0001$, compared with the differentiated sh-control group. (b, c) The density ratio of target proteins to GAPDH for 7 and 14 days. 


\section{Data Availability}

The datasets used and/or analyzed during the current study are available from the corresponding author on reasonable request.

\section{Conflicts of Interest}

The authors declare that they have no conflicts of interest.

\section{Authors' Contributions}

Ming Chen and Yeqing Yang contributed equally to this work and should be considered as co-first authors.

\section{Acknowledgments}

The authors would like to thank the School of Stomatology of Southern Medical University for kindly providing experiment platform and samples. The authors also extend special thanks to the laboratory members for their contributions. This work was supported by the General Program of National Natural Scientific Foundation of China (No. 8187041227), Medical Scientific Research Foundation of Guangdong Province of China (No. B2018012), Youth Program of President Foundation of Nanfang Hospital, Southern Medical University (No. 2016C023), and Special Program of President Foundation of Nanfang Hospital, Southern Medical University (No.2017Z004).

\section{Supplementary Materials}

Additional file 1: Table S1: the expression profiles of upregulated circRNAs in induced and noninduced hDPSCs. Additional file 2: Table S2: the expression profiles of downregulated circRNAs in induced and noninduced hDPSCs. Additional file 3: certificate of English editing. (Supplementary materials)

\section{References}

[1] G. H. Yassen, M. M. Vail, T. G. Chu, and J. A. Platt, "The effect of medicaments used in endodontic regeneration on root fracture and microhardness of radicular dentine," International Endodontic Journal, vol. 46, no. 7, pp. 688-695, 2013.

[2] R. A. Gangolli, S. M. Devlin, J. A. Gerstenhaber, P. I. Lelkes, and M. Yang, "A bilayered poly (lactic-co-glycolic acid) scaffold provides differential cues for the differentiation of dental pulp stem cells," Tissue Engineering Part A, vol. 25, no. 3-4, pp. 224-233, 2019.

[3] Q. Zhai, Z. Dong, W. Wang, B. Li, and Y. Jin, "Dental stem cell and dental tissue regeneration," Frontiers in Medicine, vol. 13, no. 2, pp. 152-159, 2019.

[4] M. Altaii, L. Richards, and G. Rossi-Fedele, "Histological assessment of regenerative endodontic treatment in animal studies with different scaffolds: a systematic review," Dental Traumatology, vol. 33, no. 4, pp. 235-244, 2017.

[5] Z. Chen, K. Zhang, W. Qiu et al., "Genome-wide identification of long noncoding RNAs and their competing endogenous RNA networks involved in the odontogenic differentiation of human dental pulp stem cells," Stem Cell Research \& Therapy, vol. 11, no. 1, p. 114, 2020.

[6] S. Yu, Y. Zhao, T. J. Fang, and L. Ge, "Effect of the Soluble Factors Released by Dental Apical Papilla-Derived Stem Cells on the Osteo/Odontogenic, Angiogenic, and Neurogenic Differentiation of Dental Pulp Cells," Stem Cells and Development, vol. 29, no. 12, pp. 795-805, 2020.

[7] F. Wang, X. Chen, Y. Han, S. Xi, and G. Wu, "CircRNA CDR1as regulated the proliferation of human periodontal ligament stem cells under a lipopolysaccharide-induced inflammatory condition," Mediators of Inflammation, vol. 2019, Article ID 1625381, 9 pages, 2019.

[8] D. Sybil, V. Jain, S. Mohanty, and S. A. Husain, "Oral stem cells in intraoral bone formation," Journal of Oral Biosciences, vol. 62, no. 1, pp. 36-43, 2020.

[9] C. Yang, R. Jia, Q. Zuo et al., "MicroRNA-143-3p regulates odontogenic differentiation of human dental pulp stem cells through regulation of the osteoprotegerin-RANK ligand pathway by targeting RANK," Experimental Physiology, vol. 105, no. 5, pp. 876-885, 2020.

[10] G. M. Ahmed, E. A. Abouauf, N. Abubakr, C. E. Dörfer, and K. F. El-Sayed, "Tissue engineering approaches for enamel, dentin, and pulp regeneration: an update," Stem Cells International, vol. 2020, Article ID 5734539, 15 pages, 2020.

[11] L. Zeng, S. Sun, L. Dong et al., "DLX3 epigenetically regulates odontoblastic differentiation of hDPCs through H19/miR-675 axis," Archives of Oral Biology, vol. 102, pp. 155-163, 2019.

[12] M. Lei, K. Li, B. Li, L. N. Gao, F. M. Chen, and Y. Jin, "Mesenchymal stem cell characteristics of dental pulp and periodontal ligament stem cells after in vivo transplantation," Biomaterials, vol. 35 , no. 24 , pp. $6332-6343,2014$.

[13] M. T. P. Albuquerque, M. C. Valera, M. Nakashima, J. E. Nör, and M. C. Bottino, "Tissue-engineering-based strategies for regenerative endodontics," Journal of Dental Research, vol. 93, no. 12, pp. 1222-1231, 2014.

[14] S.-K. Jun, J.-Y. Yoon, C. Mahapatra et al., "Ceria-incorporated MTA for accelerating odontoblastic differentiation via ROS downregulation," Dental Materials, vol. 35, no. 9, pp. 12911299, 2019.

[15] H. Tao, H. Lin, Z. Sun et al., "Klf4 promotes dentinogenesis and odontoblastic differentiation via modulation of TGF- $\beta$ signaling pathway and interaction with histone acetylation," Journal of Bone and Mineral Research, vol. 34, no. 8, pp. 1502-1516, 2019.

[16] X. Lu, X. Chen, J. Xing et al., "miR-140-5p regulates the odontoblastic differentiation of dental pulp stem cells via the Wnt $1 / \beta$-catenin signaling pathway," Stem Cell Research of Therapy, vol. 10, no. 1, p. 226, 2019.

[17] M. Guo, J. Qiu, F. Shen et al., "Comprehensive analysis of circular RNA profiles in skeletal muscles of aging mice and after aerobic exercise intervention," Aging, vol. 12, pp. 5071-5090, 2020.

[18] C. Zhang, B. Zhang, B. Yuan et al., "RNA-Seq profiling of circular RNAs in human small cell lung cancer," Epigenomics, vol. 12, no. 8, pp. 685-700, 2020.

[19] S. Qu, X. Yang, X. Li et al., "Circular RNA: a new star of noncoding RNAs," Cancer Letters, vol. 365, no. 2, pp. 141-148, 2015.

[20] W. R. Jeck, J. A. Sorrentino, K. Wang et al., "Circular RNAs are abundant, conserved, and associated with ALU repeats," RNA, vol. 19, no. 2, pp. 141-157, 2013. 
[21] J. Li, J. Yang, P. Zhou et al., "Circular RNAs in cancer: novel insights into origins, properties, functions and implications," American Journal of Cancer Research, vol. 5, no. 2, pp. 472480, 2015.

[22] F. Wang, A. J. Nazarali, and S. Ji, "Circular RNAs as potential biomarkers for cancer diagnosis and therapy," American Journal of Cancer Research, vol. 6, no. 6, pp. 1167-1176, 2016.

[23] S. Li, C. Lin, J. Zhang et al., "Quaking promotes the odontoblastic differentiation of human dental pulp stem cells," Journal of Cellular Physiology, vol. 233, no. 9, pp. 7292-7304, 2018.

[24] X. Gu, M. Li, Y. Jin, D. Liu, and F. Wei, "Identification and integrated analysis of differentially expressed lncRNAs and circRNAs reveal the potential ceRNA networks during PDLSC osteogenic differentiation," BMC Genet, vol. 18, no. 1, p. 100, 2017.

[25] L. Wang, Y. Wei, Y. Yan et al., "CircDOCK1 suppresses cell apoptosis via inhibition of miR-196a-5p by targeting BIRC3 in OSCC," Oncology Reports, vol. 39, pp. 951-966, 2017.

[26] X. Li, Y. Zheng, Y. Zheng et al., "Circular RNA CDR1as regulates osteoblastic differentiation of periodontal ligament stem cells via the miR-7/GDF5/SMAD and p38 MAPK signaling pathway," Stem Cell Research \& Therapy, vol. 9, no. 1, p. 232, 2018.

[27] Z. Li, N. Li, X. Ge et al., "Differential circular RNA expression profiling during osteogenic differentiation of stem cells from apical papilla," Epigenomics, vol. 11, no. 9, pp. 1057-1073, 2019.

[28] V. Mayo, Y. Sawatari, C. Y. C. Huang, and F. Garcia-Godoy, "Neural crest-derived dental stem cells - where we are and where we are going," Journal of Dentistry, vol. 42, no. 9, pp. 1043-1051, 2014.

[29] W. Jiang, H. Lv, H. Wang et al., "Activation of the NLRP3/caspase-1 inflammasome in human dental pulp tissue and human dental pulp fibroblasts," Cell and Tissue Research, vol. 361, no. 2, pp. 541-555, 2015.

[30] X. Wei, J. Ling, L. Wu, L. Liu, and Y. Xiao, "Expression of mineralization markers in dental pulp cells," Journal of Endodontia, vol. 33, no. 6, pp. 703-708, 2007.

[31] F. Langenbach and J. Handschel, "Effects of dexamethasone, ascorbic acid and $\beta$-glycerophosphate on the osteogenic differentiation of stem cells in vitro," Stem Cell Research and Therapy, vol. 4, no. 5, p. 117, 2013.

[32] L. Chen, Z. Song, S. Huang et al., "IncRNA DANCR suppresses odontoblast-like differentiation of human dental pulp cells by inhibiting wnt/ $\beta$-catenin pathway," Cell and Tissue Research, vol. 364, no. 2, pp. 309-318, 2016.

[33] H. Salkin, Z. B. Gönen, E. Ergen, D. Bahar, and M. Çetin, "Effects of TGF- $\beta 1$ overexpression on biological characteristics of human dental pulp-derived mesenchymal stromal cells," International Journal of Stem Cells, vol. 12, no. 1, pp. 170-182, 2019.

[34] S. H. Z. Ariffin, S. Kermani, R. M. A. Wahab, S. Senafi, Z. Z. Ariffin, and M. A. Razak, "In vitro chondrogenesis transformation study of mouse dental pulp stem cells," The Scientific World Journal, vol. 2012, Article ID 827149, 7 pages, 2012.

[35] K. J. Livak and T. D. Schmittgen, "Analysis of relative gene expression data using real-time quantitative PCR and the 2$\Delta \Delta$ CT method," Methods, vol. 25, no. 4, pp. 402-408, 2001.

[36] D. J. Hunter, C. Bardet, S. Mouraret et al., "Wnt Acts as a Prosurvival Signal to Enhance Dentin Regeneration," Journal of
Bone and Mineral Research, vol. 30, no. 7, pp. 1150-1159, 2015.

[37] S. Oka, K. Oka, X. Xu, T. Sasaki, P. Bringas, and Y. Chai, "Cell autonomous requirement for TGF- $\beta$ signaling during odontoblast differentiation and dentin matrix formation," Mechanisms of Development, vol. 124, no. 6, pp. 409-415, 2007.

[38] C. Li and H. Jiang, "Altered expression of circular RNA in human dental pulp cells during odontogenic differentiation," Molecular Medicine Reports, vol. 20, pp. 871-878, 2019.

[39] A. H. De-La-Cruz-Montoya, E. G. Ramírez-Salazar, M. M. Martínez-Aguilar et al., "Identification of miR-708-5p in peripheral blood monocytes: potential marker for postmenopausal osteoporosis in Mexican-Mestizo population," Experimental Biology and Medicine, vol. 243, no. 13, pp. 10271036, 2018.

[40] R. Wu, J. Ruan, Y. Sun et al., "Long non-coding RNA HIF1AAS2 facilitates adipose-derived stem cells (ASCs) osteogenic differentiation through miR-665/IL6 axis via PI3K/Akt signaling pathway," Stem Cell Research \& Therapy, vol. 9, no. 1, p. $348,2018$.

[41] H. M. Heair, A. G. Kemper, B. Roy et al., "MicroRNA-665 regulates dentinogenesis through microRNA-mediated silencing and epigenetic mechanisms," Molecular and Cellular Biology, vol. 35, pp. 3116-3130, 2015.

[42] M. Elsafadi, M. Manikandan, N. M. Alajez et al., "MicroRNA4739 regulates osteogenic and adipocytic differentiation of immortalized human bone marrow stromal cells via targeting LRP3,” Stem Cell Research, vol. 20, pp. 94-104, 2017.

[43] V. Chrepa, M. A. Henry, B. J. Daniel, and A. Diogenes, "Delivery of apical mesenchymal stem cells into root canals of mature teeth," Journal of Dental Research, vol. 94, no. 12, pp. 1653-1659, 2015.

[44] H. Shetty, S. Shetty, A. Kakade, R. Desai, C. F. Zhang, and P. Neelakantan, "Cone-beam computed tomographic and histological investigation of regenerative endodontic procedure in an immature mandibular second premolar with chronic apical abscess," Journal of Investigative and Clinical Dentistry, vol. 9, no. 4, p. e12352, 2018

[45] E. Piva, S. A. Tarlé, J. E. Nör et al., "Dental pulp tissue regeneration using dental pulp stem cells isolated and expanded in human serum," Journal of Endodontia, vol. 43, no. 4, pp. 568-574, 2017.

[46] M. Torabinejad, M. Milan, S. Shabahang, K. R. Wright, and H. Faras, "Histologic examination of teeth with necrotic pulps and periapical lesions treated with 2 scaffolds: an animal investigation," Journal of Endodontia, vol. 41, no. 6, pp. 846-852, 2015.

[47] A. Diogenes, N. B. Ruparel, Y. Shiloah, and K. M. Hargreaves, "Regenerative endodontics a way forward," The Journal of the American Dental Association, vol. 147, no. 5, pp. 372-380, 2016. 